\title{
STABILITY OF FRONT SOLUTIONS IN A MODEL FOR A SURFACTANT DRIVEN FLOW ON AN INCLINED PLANE
}

\author{
Anna Ghazaryan ${ }^{\text {a }}$, Stéphane Lafortune ${ }^{\mathrm{b}}$, Vahagn Manukian ${ }^{\mathrm{c}}$ \\ ${ }^{a}$ Department of Mathematics, Miami University, Bachelor Hall, Oxford, OH 45056, USA, Ph. 1-513-785- \\ 3220, ghazarar@miamioh.edu \\ b Department of Mathematics, College of Charleston, 175 Calhoun Street, Charleston, SC 29401, USA, Ph. \\ 1-843-953-5869, lafortunes@cofc.edu \\ ${ }^{\mathrm{c}}$ Department of Mathematics, Miami University, 1601 University Blvd, Hamilton, OH 45011, USA, Ph. \\ 1-513-785-3220, manukive@miamioh.edu
}

\begin{abstract}
We consider a model for the flow of a thin liquid film down an inclined plane in the presence of a surfactant. The model is known to possess various families of traveling wave solutions. We use a combination of analytical and numerical methods to study the stability of the traveling waves. We show that for at least some of these waves the spectra of the linearization of the system about them are within the closed left-half complex plane.
\end{abstract}

Keywords. traveling waves, stability of traveling waves, Evans function, surfactants, parabolic systems.

AMS Subject Classification. 35B35, 35B36, 34C37, 76D08.

\section{INTRODUCTION}

In this paper we analyze a model that describes the flow of thin liquid film down the inclined plane, which is modified by the presence of insoluble surfactant, and with the effects of gravity taken into account $[10,11]$. Surfactants as media that stays on the surface of a thin film flow have a variety of applications, from industrial to medical $[14,17,18,27]$. Rooted in lubrication theory [10,11], the model consists of a system of nonlinearly coupled partial differential equations

$$
\begin{aligned}
& h_{t}-\frac{1}{2}\left(h^{2} \Gamma_{x}\right)_{x}+\frac{\alpha}{3}\left(h^{3}\right)_{x}=\frac{\beta}{3}\left(h^{3} h_{x}\right)_{x}, \\
& \Gamma_{t}-\left(h \Gamma \Gamma_{x}\right)_{x}+\frac{\alpha}{2}\left(h^{2} \Gamma\right)_{x}=\frac{\beta}{2}\left(h^{2} h_{x} \Gamma\right)_{x}+D \Gamma_{x x},
\end{aligned}
$$

where $h(x, t)$ represents the hight of the thin film, $\Gamma(x, t)$ represents the surfactant concentration at time $t$ and $x$ is the space variable along the inclined plane. Parameter $D$ is proportional to the inverse of the Péclet number and acts as a diffusion constant of the surfactant concentration. Péclet number measures the relative contribution of mass transport by diffusion against mass transport by advection. Smaller values of $D$, which we assume positive, indicate the larger influence of advection, larger ones indicate the stronger influence of the diffusion. Parameters $\alpha$ and $\beta$ encode the steepness of the incline: $\alpha$ is proportional to the sine of the angle formed by the inclined plane with the horizontal surface, and $\beta$ is proportional to the cosine of the same angle. This model takes into account the Marangoni force, which is due to the modification of the surface tension by the presence of a surfactant.

Date: April 30, 2015. 
The system (1.1) is a limiting system for

$$
\begin{aligned}
& h_{t}-\frac{1}{2}\left(h^{2} \Gamma_{x}\right)_{x}+\frac{\alpha}{3}\left(h^{3}\right)_{x}=-\frac{C}{3}\left(h^{3} h_{x x x}\right)_{x}+\frac{\beta}{3}\left(h^{3} h_{x}\right)_{x}, \\
& \Gamma_{t}-\left(h \Gamma \Gamma_{x}\right)_{x}+\frac{\alpha}{2}\left(h^{2} \Gamma\right)_{x}=-\frac{C}{2}\left(h^{2} h_{x x x} \Gamma\right)_{x}+\frac{\beta}{2}\left(h^{2} h_{x} \Gamma\right)_{x}+D \Gamma_{x x},
\end{aligned}
$$

as $C \rightarrow 0$, where $C$ is a quantity proportional to the capillary number. The system (1.2) is obtained from the two-dimensional Navier-Stokes equation in $[11,21,27,38]$ and the existence of traveling wave solutions that connect two different constant states have been studied in [29, 30,32]. In [29, 30], the authors consider the case where at least one of the parameters is zero, while [32] studies different parameter regimes when all the parameters are positive.

The stability of traveling waves very often is of critical importance. For example, in surfactant replacement therapy a coating of surfactant is used to support a healthy lung function [20]. The development of stable wavefronts is related to the mechanism of the surfactant delivery for the lung.

Numerical simulations performed in [30] suggest that in some parameter regimes traveling wave solutions are stable. It is also mentioned in [30] that the analysis of the stability of the individual waves of (1.2) when all parameters are nonzero is of interest for applications. In this paper we study the stability of wavefronts of the limiting for (1.2) system (1.1). The stability of these wavefronts will factor in the stability analysis of wavefronts in the full system (1.2) through the multi-scale approach. The latter will be a subject of future work.

We note that the parameter $\alpha$ can be normalized to be 1 in the above equations by rescaling

$$
\bar{x}=\alpha x, \quad \bar{t}=\alpha^{2} t,
$$

and dropping the bars as in [32]. After this transformation, the system (1.1) reads

$$
\begin{aligned}
& h_{t}-\frac{1}{2}\left(h^{2} \Gamma_{x}\right)_{x}+\frac{1}{3}\left(h^{3}\right)_{x}=\frac{\beta}{3}\left(h^{3} h_{x}\right)_{x}, \\
& \Gamma_{t}-\left(h \Gamma \Gamma_{x}\right)_{x}+\frac{1}{2}\left(h^{2} \Gamma\right)_{x}=\frac{\beta}{2}\left(h^{2} h_{x} \Gamma\right)_{x}+D \Gamma_{x x} .
\end{aligned}
$$

Traveling waves are sought as stationary solutions of the form $(h(\xi), \Gamma(\xi))$, where $\xi=x-s t$ is the traveling wave coordinate, and $s$ is an undetermined at the moment parameter related to the speed of the wave, so $(h(\xi), \Gamma(\xi))$ solves the following system of ordinary differential equations

$$
\begin{aligned}
& s h_{\xi}-\frac{1}{2}\left(h^{2} \Gamma_{\xi}\right)_{\xi}+\frac{1}{3}\left(h^{3}\right)_{\xi}=\frac{\beta}{3}\left(h^{3} h_{\xi}\right)_{\xi}, \\
& s \Gamma_{\xi}-\left(h \Gamma \Gamma_{x}\right)_{\xi}+\frac{1}{2}\left(h^{2} \Gamma\right)_{\xi}=\frac{\beta}{2}\left(h^{2} h_{\xi} \Gamma\right)_{\xi}+D \Gamma_{\xi \xi} .
\end{aligned}
$$

To capture wavefronts in this system one imposes boundary-like conditions

$$
h(-\infty)=h_{L}, \quad h(+\infty)=h_{R}, \quad \Gamma( \pm \infty)=0,
$$

which indicate that we are interested in traveling waves that are shaped as a front in the $h$-component and as a pulse in $\Gamma$-component. With these boundary condition, integration of (1.4) leads to

$$
\begin{aligned}
& -h s-\frac{1}{2} h^{2} \Gamma^{\prime}+\frac{1}{3} h^{3}=\frac{\beta}{3} h^{3} h^{\prime}+K_{1}, \\
& -\Gamma s-h \Gamma \Gamma^{\prime}+\frac{1}{2} h^{2} \Gamma=\frac{\beta}{2} h^{2} h^{\prime} \Gamma+D \Gamma^{\prime},
\end{aligned}
$$

where the derivative is taken with respect to $\xi$, and the quantity

$$
K_{1}=-\frac{1}{3} h_{L} h_{R}\left(h_{L}+h_{R}\right)
$$

is the constant of integration expressed through $h_{L}$ and $h_{R}$. 
The wave speed is related to the boundary values as

$$
s=\frac{1}{3}\left(h_{L}^{2}+h_{L} h_{R}+h_{R}^{2}\right) .
$$

The system (1.6) can be simplified by replacing the second equation with a linear combination of the first and second equations with respective coefficients $-\frac{2}{h}$ and $\frac{2}{\Gamma}$ and written as a first order system

$$
\begin{aligned}
h^{\prime} & =\frac{1}{\beta h^{3}}\left(h^{3}-3 s h-3 K_{1}-3 h \Gamma \frac{s h+3 K_{1}}{h \Gamma+4 D}\right), \\
\Gamma^{\prime} & =\frac{2 \Gamma}{h} \frac{s h+3 K_{1}}{h \Gamma+4 D} .
\end{aligned}
$$

We assume as in $[30]$ that

$$
0<\frac{h_{R}}{h_{L}}<\frac{\sqrt{3}-1}{2}
$$

where $h_{L}$ and $h_{R}$ are the positive roots of the polynomial $h^{3}-3 s h-3 K_{1}$, which can be factored as

$$
h^{3}-3 s h-3 K_{1}=\left(h-h_{L}\right)\left(h-h_{R}\right)\left(h+h_{L}+h_{R}\right) .
$$

Then, the traveling wave solutions correspond to the heteroclinic connections that asymptotically connect $(h, \Gamma)=\left(h_{R}, 0\right)$ to $(h, \Gamma)=\left(h_{L}, 0\right)$. An example of such heteroclinic connection, together with the traveling wave it represents, is shown in Figure 4.1. The existence of continuum of such solutions (parametrized by the maximum value of $\Gamma$ ) is shown in [32] (more specifically, see sections called Region 2, Region 4, and Region 5 in [32]). In [32], the system (1.9) represents the reduced flow on a normally hyperbolic invariant manifold description of which is obtained by exploiting the multi-scale structure of the full system (1.2) when the parameter $C$ is small.

We point out that the line $\Gamma=0$ is an invariant set for the flow induced by the system (1.9), and there is an asymptotic connection between $\left(h_{L}, 0\right)$ to $\left(h_{R}, 0\right)$ along the set $\Gamma=0$.

The main ingredient of the stability analysis of a traveling wave is to find the location of the spectrum of the linearization of the pde system (1.3) about the traveling wave. The presence of spectra with positive real parts indicates an instability as perturbations to the wave then grow in amplitude at exponential rates. Spectrum on the imaginary axis indicates that the perturbations may not decay. In this paper, in Sect. 2 we analytically prove that the linearization of the pde system (1.3) about the wavefront with $\Gamma=0$ does not have spectrum with nonnegative real parts, with the exception of the origin. We also perform Evans function numerical computations (Sect. 4) combined with energy estimates (Sect. 3) which show that some of the wavefronts with $\Gamma \neq 0$ do not have spectrum within the closed right half of complex plane, with the exception of the spectrum at the origin.

Let $(h, \Gamma)$ be traveling wave solutions of (1.6). The linearization of the pde system (1.3) around the traveling wave solutions $(h, \Gamma)$ gives rise to the following eigenvalue problem

$$
\begin{aligned}
& \lambda V=\left(s V+\frac{1}{2}\left(2 h \Gamma_{\xi} V+h^{2} U_{\xi}\right)-h^{2} V+\frac{\beta}{3}\left(3 h^{2} h_{\xi} V+h^{3} V_{\xi}\right)\right)_{\xi}, \\
& \lambda U=\left(s U+\Gamma \Gamma_{\xi} V+h \Gamma_{\xi} U+h \Gamma U_{\xi}-h \Gamma V-\frac{1}{2} h^{2} U+\frac{\beta}{2}\left(2 h \Gamma h_{\xi} V+h^{2} h_{\xi} U+h^{2} \Gamma V_{\xi}\right)+D U_{\xi}\right)_{\xi} .
\end{aligned}
$$

In the following sections we study the eigenvalue problem (1.12) along with the traveling wave system (1.9). 


\section{Spectrum in the CASE Where $\Gamma=0$.}

2.1. Formulation of the result and the setting of the problem. In this section we investigate the spectrum of the linearization (1.12) at the traveling wave $(h, \Gamma=0)$. We consider (1.12) with $\Gamma=0$,

$$
\begin{aligned}
& \lambda V=\left(s V+\frac{1}{2} h^{2} U_{\xi}-h^{2} V+\frac{\beta}{3}\left(3 h^{2} h_{\xi} V+h^{3} V_{\xi}\right)\right)_{\xi}, \\
& \lambda U=\left(\left(s-\frac{1}{2} h^{2}+\frac{\beta}{2} h^{2} h_{\xi}\right) U+D U_{\xi}\right)_{\xi},
\end{aligned}
$$

coupled with

$$
h_{\xi}=\frac{1}{\beta h^{3}}\left(h^{3}-3 s h-3 K_{1}\right) .
$$

Combining (2.2) and (2.1) allows us to obtain the system

$$
\begin{aligned}
& \lambda V=\left(-\frac{2 h s+3 K_{1}}{h} V+\frac{1}{2} h^{2} U_{\xi}+\frac{\beta}{3} h^{3} V_{\xi}\right)_{\xi}, \\
& \lambda U=\left(-\frac{h s+3 K_{1}}{2 h} U+D U_{\xi}\right)_{\xi} .
\end{aligned}
$$

We do not have an explicit expression for $h(\xi)$ as a function of $\xi$, however, below we obtain the critical information we need for our analyses from (2.2). We note that the equation (2.2) plays a role of the compactification used in [2].

The second equation in (2.3) is decoupled from the first one and can be written as

$$
D U_{\xi \xi}+(f(h) U)_{\xi}=\lambda U
$$

where

$$
f(h)=-\frac{s h+3 K_{1}}{2 h} .
$$

Then the eigenvalue problem (2.4) can be cast as

$$
\frac{1}{r_{U}(\xi)}\left(\frac{\mathrm{d}^{2}}{\mathrm{~d} \xi^{2}} U+p_{U}(\xi) \frac{\mathrm{d}}{\mathrm{d} \xi} U+q_{U}(\xi) U\right)=\lambda U
$$

where

$$
\begin{aligned}
r_{U}(\xi) & =\frac{1}{D} \\
p_{U}(\xi) & =\frac{f(h(\xi))}{D}=-\frac{s h(\xi)+3 K_{1}}{2 D h(\xi)}
\end{aligned}
$$

and

$$
q_{U}(\xi)=\frac{1}{D} \frac{\mathrm{d}}{\mathrm{d} \xi} f(h(\xi))=\frac{3 K_{1}}{2 D h^{2}(\xi)} h_{\xi}(\xi)=\frac{3 K_{1}}{2 \beta D h^{5}(\xi)}\left(h^{3}(\xi)-3 \operatorname{sh}(\xi)-3 K_{1}\right) .
$$

Similarly, we can rewrite the first equation in (2.3) as

$$
\frac{1}{r_{V}(\xi)}\left(\frac{d^{2}}{d \xi^{2}} V+p_{V}(\xi) \frac{d}{d \xi} V+q_{V}(\xi) V\right)+a_{V}(\xi) \frac{d^{2}}{d \xi^{2}} U+b_{V}(\xi) \frac{d}{d \xi} U=\lambda V
$$


where

$$
\begin{aligned}
r_{V}(\xi) & =\frac{3}{\beta h(\xi)^{3}} \\
p_{V}(\xi) & =3 \frac{h^{3}(\xi)-3 \operatorname{sh}(\xi)-3 K_{1}-\left(2 s h(\xi)+3 K_{1}\right)}{\beta h^{4}(\xi)} \\
q_{V}(\xi) & =\frac{9 K_{1}}{\beta^{2} h^{8}(\xi)}\left(h^{3}(\xi)-3 \operatorname{sh}(\xi)-3 K_{1}\right) \\
a_{V}(\xi) & =\frac{h^{2}(\xi)}{2} \\
b_{V}(\xi) & =\frac{1}{\beta h^{2}(\xi)}\left(h^{3}(\xi)-3 \operatorname{sh}(\xi)-3 K_{1}\right) .
\end{aligned}
$$

The system (2.3) then can thus be written in a matrix form

$$
\mathcal{L} w=\lambda w
$$

where

$$
\mathcal{L}=\left(\begin{array}{cc}
\mathcal{L}_{V} & \mathcal{L}_{m} \\
0 & \mathcal{L}_{U}
\end{array}\right), \quad w=\left(\begin{array}{c}
V \\
U
\end{array}\right)
$$

and

$$
\begin{aligned}
\mathcal{L}_{U} & =\frac{1}{r_{U}(\xi)}\left(\frac{\mathrm{d}^{2}}{\mathrm{~d} \xi^{2}}+p_{U}(\xi) \frac{\mathrm{d}}{\mathrm{d} \xi}+q_{U}(\xi)\right), \\
\mathcal{L}_{V} & =\frac{1}{r_{V}(\xi)}\left(\frac{d^{2}}{d \xi^{2}}+p_{V}(\xi) \frac{d}{d \xi}+q_{V}(\xi)\right), \\
\mathcal{L}_{m} & =a_{V}(\xi) \frac{d^{2}}{d \xi^{2}}+b_{V}(\xi) \frac{d}{d \xi} .
\end{aligned}
$$

The spectrum of the operator $\mathcal{L}$ has two parts: the essential spectrum that is due to the behavior of the constants states of the wavefront solution and the point spectrum. In this section, we shall prove the following theorem.

Theorem 2.1. The spectrum of the operator $\mathcal{L}$ considered on $L^{2}(\mathbb{R}) \times L^{2}(\mathbb{R})$ belongs to $\{\operatorname{Re}(\lambda)<0\} \cup\{0\}$.

Since $\mathcal{L}$ has an upper diagonal structure, then if $\lambda$ is an eigenvalue of $\mathcal{L}$ and is associated with an eigenvector $\left(V_{\lambda}(\xi), U_{\lambda}(\xi)\right)$, where $U_{\lambda}(\xi)$ is not identically zero, then the eigenvalue $\lambda$ is also an eigenvalue of $\mathcal{L}_{U}$ with associated eigenfunction $U_{\lambda}(\xi)$. Moreover, it is also true that if $\lambda$ is an eigenvalue of $\mathcal{L}_{U}$ with associated eigenvector $U_{\lambda}(\xi)$, then $\lambda$ is an eigenvalue of $\mathcal{L}$ associated with an eigenvector $\left(0, U_{\lambda}(\xi)\right)$. We show that the operator $\mathcal{L}$ does not have positive eigenvalues by showing at first that $\mathcal{L}_{U}$ does not have eigenvalues with positive real part, then it follows from the structure of $\mathcal{L}$, if $\lambda$ is an eigenvalue of $\mathcal{L}$ with positive real part, then the associated eigenvector has the form $\left(V_{\lambda}(\xi), 0\right)$. Therefore, in order to show that the eigenvalue problem (2.8) does not have a positive eigenvalue, we show that the operators $\mathcal{L}_{U}$ and $\mathcal{L}_{V}$ do not have unstable point spectrum, that is the eigenvalue problems

$$
\frac{\mathrm{d}^{2}}{\mathrm{~d} \xi^{2}} S+p_{S}(\xi) \frac{\mathrm{d}}{\mathrm{d} \xi} S+q_{S}(\xi) S=\lambda r_{S}(\xi) S, \quad S=U \text { or } V,
$$

where the subscript $S$ indicates whether this equation is related to the first or the second equation in the system (2.3), does not have an eigenvalue with positive real part. Since $r_{U}(\xi)$ and $r_{V}(\xi)$ are positive when $\xi \in(-\infty,+\infty)$, and have continuous second derivatives, and $p_{U}(\xi)$ and $p_{V}(\xi)$ have continuous first 
derivatives, therefore we use the Liouville transformation for both $S=U$ and $S=V$,

$$
\begin{aligned}
\zeta & =\int_{\xi_{S}^{*}}^{\xi} \sqrt{r_{S}(t)} d t, \\
S(\xi) & =\rho_{S}^{-\frac{1}{2}}(\xi) \eta_{S}(\zeta),
\end{aligned}
$$

where

$$
\rho_{S}(\xi)=\left(r_{S}(\xi)\right)^{\frac{1}{2}} \exp \left(\int_{\xi_{S}^{*}}^{\xi} p_{S}(\xi) d \xi\right)
$$

and $\xi_{S}^{*} \in \mathbb{R}$ is any constant. This transformation reduces the eigenvalue problem (2.11) to

$$
\mathcal{L}_{S}^{\rho} \eta_{S}=\lambda \eta_{S}
$$

where

$$
\mathcal{L}_{S}^{\rho}=\frac{\mathrm{d}^{2}}{\mathrm{~d} \xi^{2}}+Q_{S}^{\rho}(\xi)
$$

and

$$
Q_{S}^{\rho}(\zeta)=\frac{q_{S}(\xi)}{r_{S}(\xi)}-\frac{1}{4} \frac{\left(p_{S}(\xi)\right)^{2}}{r_{S}(\xi)}-\frac{1}{2} \frac{p_{S}^{\prime}(\xi)}{r_{S}(\xi)}-\frac{1}{4} \frac{r_{S}^{\prime \prime}(\xi)}{\left(r_{S}(\xi)\right)^{2}}+\frac{5}{16} \frac{\left(r_{S}^{\prime}(\xi)\right)^{2}}{\left(r_{S}(\xi)\right)^{3}}
$$

Note that $Q_{\eta}^{S}(\zeta)$ is given explicitly in the original variable $\xi$. Since $r_{S}(\xi)>0$, the first relation in $(2.12)$ corresponds to the reparameterization of the space variable, while the second relation puts (2.11) in the canonical form (2.13) in new variable $\eta_{S}(\xi)$ (see [28]).

In the next section we study the spectrum of $\mathcal{L}_{S}^{\rho}$ and relate the obtained information to the spectrum of the operator $\mathcal{L}_{S}$ that is defined in (2.10) for $S=U$ or $S=V$.

2.2. Analyses of eigenvalue problems (2.11). We transform the second equation of the eigenvalue problem (2.3) using the coordinate transformation

$$
U=U, \quad U_{1}=D U^{\prime}-\frac{s h+3 K_{1}}{2 h} U,
$$

to a first order system and couple it to the traveling wave ordinary differential equation (2.2), thus, obtaining

$$
\begin{aligned}
D U^{\prime} & =U_{1}+\frac{s h+3 K_{1}}{2 h} U, \\
U_{1}^{\prime} & =\lambda U, \\
h^{\prime} & =\frac{1}{\beta h^{3}}\left(h^{3}-3 s h-3 K_{1}\right),
\end{aligned}
$$

where the prime corresponds to the derivative with respect to $\xi$. Equations (2.16) are equivalent to (2.4), or (2.11) with $S=U$.

First, we show that $\lambda=0$ is an eigenvalue of (2.16). Since we are looking for a solution that satisfy $U_{1}( \pm \infty)=0$, then $\lambda=0$ implies that $U_{1}(\xi)$ has to be identically zero. When $U_{1}(\xi) \equiv 0$, the reduced system

$$
\begin{aligned}
D U^{\prime} & =\frac{s h+3 K_{1}}{2 h} U, \\
h^{\prime} & =\frac{1}{\beta h^{3}}\left(h^{3}-3 s h-3 K_{1}\right)
\end{aligned}
$$

has two fixed points: $(U, h)=\left(0, h_{\mathrm{L}}\right)$ and $(U, h)=\left(0, h_{\mathrm{R}}\right)$. From the flow of $(2.17)$ it follows that there is a solution that asymptotically connects these two fixed points which is unique up to a multiplicative constant. This nontrivial solution can be expressed as

$$
U_{0}(\xi)=K_{U} \exp \left(\int_{\xi_{U}^{*}}^{\xi} \frac{\operatorname{sh}(\tau)+3 K_{1}}{2 D h(\tau)} d \tau\right),
$$


where $K_{U}$ is an arbitrary nonzero constant, and $\xi_{U}^{*}$ is a zero of $p_{U}(\xi)$, or, alternatively, a zero of $f(h(\xi))$ (see $(2.5))$. The expression (2.18) represents the eigenfunction $U_{0}(\xi)$ associated with the eigenvalue $\lambda=0$. From (2.18) and also from (2.17) it is evident that $U_{0}(\xi)$ does not have any zeros, which is a piece of information that we use below to deduce that the operator $\mathcal{L}_{U}$ does not contribute an unstable eigenvalue to the spectrum of $\mathcal{L}$.

Next we write the eigenvalue problem (2.4) as

$$
\frac{\mathrm{d}^{2}}{\mathrm{~d} \xi^{2}} U+p_{U}(\xi) \frac{\mathrm{d}}{\mathrm{d} \xi} U+q_{U}(\xi) U=\frac{\lambda}{D} U
$$

Recall that

$$
\begin{aligned}
p_{U}(\xi) & =-\frac{s h(\xi)+3 K_{1}}{2 D h(\xi)}, \\
q_{U}(\xi) & =\frac{3 K_{1}}{2 \beta D h^{5}(\xi)}\left(h^{3}(\xi)-3 \operatorname{sh}(\xi)-3 K_{1}\right) .
\end{aligned}
$$

Note that when $D \neq 0,(2.19)$ can be seen as an eigenvalue problem with an eigenvalue $\Lambda=\frac{\lambda}{D}$.

Since $h(\xi)>0$ and smooth, the functions $p_{U}(\xi)$ and $q_{U}(\xi)$ are smooth functions on $\mathbb{R}$ with the following properties: the limits below exist

$$
\begin{array}{r}
p_{U}^{+}=\lim _{\xi \rightarrow \infty} p_{U}(\xi)=-\lim _{\xi \rightarrow \infty} \frac{s h(\xi)+3 K_{1}}{2 D h(\xi)}=-\frac{s h_{R}+3 K_{1}}{2 D h_{R}}=\frac{1}{6 D}\left(2 h_{L}^{2}+2 h_{L} h_{R}-h_{R}^{2}\right), \\
p_{U}^{-}=\lim _{\xi \rightarrow-\infty} p_{U}(\xi)=-\lim _{\xi \rightarrow \infty} \frac{s h(\xi)+3 K_{1}}{2 D h(\xi)}=-\frac{s h_{L}+3 K_{1}}{2 D h_{L}}=\frac{1}{6 D}\left(2 h_{R}^{2}+2 h_{L} h_{R}-h_{L}^{2}\right),
\end{array}
$$

and

$$
q_{U}^{ \pm}=\lim _{\xi \rightarrow \pm \infty} q_{U}(\xi)=0 .
$$

The convergence of the limits is at the same exponential rate as for $h(\xi)$ converging to constant states $h_{L}$ and $h_{R}$ at $\pm \infty$ correspondingly. Note that $p_{U}(\xi)$ becomes zero for some value of $\xi=\xi_{U}^{*}$ which corresponds to a zero of $f(h)$,

$$
f\left(h^{*}\right)=0 \text { for } h^{*}=\frac{3 h_{L} h_{R}\left(h_{L}+h_{R}\right)}{h_{R}^{2}+h_{L} h_{R}+h_{L}^{2}} .
$$

From the expression for $h^{*}$ and the assumption (1.11) on $h_{L}$ and $h_{R}$, one can conclude that $h_{R}<h^{*}<h_{L}$. The operator $\mathcal{L}_{U}$ is self-adjoint in the inner product weighted by

$$
\rho_{U}(\xi)=\exp \left(\int_{\xi_{U}^{*}}^{\xi} p_{U}(\tau) d \tau\right)=\exp \left(\int_{\xi_{U}^{*}}^{\xi}-\frac{s h(\tau)+3 K_{1}}{2 D h(\tau)} d \tau\right) .
$$

By making the substitution $u=h(\tau)$ in $(2.23)$ and using the fact that $h(\xi)$ solves the traveling wave equation (2.2) we can relate the weight to $h$ as

$$
\rho_{U}(\xi)=\exp \left(\int_{h_{*}}^{h(\xi)}-\frac{\beta u^{2}}{2 D} \frac{s u+3 K_{1}}{u^{3}-3 s u-3 K_{1}} d u\right) .
$$

Since the integrand in (2.24) is a rational function where the denominator can be written as a product of linear factors, we integrate it and express $\rho_{U}(\xi)$ as

$$
\rho_{U}(\xi)=C(h(\xi))\left(h(\xi)-h_{R}\right)^{-\frac{\beta h_{R}^{3}}{6 D} \frac{2 h_{L}^{2}+2 h_{L} h_{R}-h_{R}^{2}}{\left(h_{L}+2 h_{R}\right)\left(h_{L}-h_{R}\right)}}\left(h(\xi)-h_{L}\right)^{-\frac{\beta h_{L}^{3}}{6 D} \cdot \frac{h_{L}^{2}-2 h_{L} h_{R}-2 h_{R}^{2}}{\left(2 h_{L}+h_{R}\right)\left(h_{L}-h_{R}\right)}},
$$

where

$$
C(h(\xi))=K\left(h_{*}\right) e^{-\frac{\beta h(\xi)}{6 D}\left(h_{L}^{2}+h_{L} h_{R}+h_{R}^{2}\right)}\left(h(\xi)+h_{L}+h_{R}\right)^{-\frac{\beta}{6 D} \frac{\left(h_{L}^{2}+4 h_{L} h_{R}+h_{R}^{2}\right)\left(h_{L}+h_{R}\right)^{3}\left(h_{L}-h_{R}\right)+2 h_{L}^{6}}{\left(h_{L}+2 h_{R}\right)\left(2 h_{L}+h_{R}\right)\left(h_{L}-h_{R}\right)}} .
$$


Since

$$
h(\xi)-h_{R}=O\left(e^{\frac{2 h_{R}^{2}-h_{L} h_{R}-h_{L}^{2}}{\beta h_{R}^{3}} \xi}\right) \quad \text { as } \quad \xi \rightarrow \infty
$$

and

$$
h(\xi)-h_{L}=O\left(e^{\frac{2 h_{L}^{2}-h_{L} h_{R}-h_{R}^{2}}{\beta h_{L}^{3}} \xi}\right) \text { as } \quad \xi \rightarrow-\infty,
$$

then from (2.25), (2.26), (2.27) and (2.28) it follows that the limits

$$
\rho_{U}^{ \pm}=\lim _{\xi \rightarrow \pm \infty} e^{-p_{U}^{ \pm} \xi} \rho_{U}(\xi)
$$

are finite. Then we use the Liouville transformation (2.12)

$$
U(\xi)=\left(\rho_{U}(\xi)\right)^{-\frac{1}{2}} \eta(\xi), \quad \xi=\frac{1}{D} x,
$$

to write the eigenvalue problem (2.4) as

$$
\eta_{U}^{\prime \prime}(\xi)+\left(Q_{U}(\xi)-\lambda\right) \eta_{U}(\xi)=0,
$$

where

$$
Q_{U}(\xi)=D\left(q_{U}(\xi)-\frac{1}{4}\left(p_{U}(\xi)\right)^{2}-\frac{1}{2} p_{U}^{\prime}(\xi)\right)
$$

and $D$ is a positive constant. In $\eta_{U}(\xi)$ variable, where

$$
\eta_{U}(\xi)=\sqrt{\rho_{U}(\xi)} U(\xi)
$$

and $x=D \xi$, the eigenvalue problem (2.31) is self adjoint in $L^{2}(\mathbb{R})$ and it follows from (2.18) and (2.32) that $\lambda=0$ is an eigenvalue of (2.31) with the eigenfunction

$$
\eta_{U}^{0}(\xi)=K \exp \left(\frac{1}{2} \int_{\xi_{U}^{*}}^{D \xi} \frac{\operatorname{sh}(\tau)+3 K_{1}}{2 D h(\tau)} d \tau\right) .
$$

The latter does not have zeros. It follows from [25, Theorem 2.3.3] that the equation (2.31) does not possess positive eigenvalues. Due to the limits $(2.21),(2.22)$ and $(2.29)$, if $\mathcal{L}_{U}$ have unstable eigenvalues, the multiplication by $\sqrt{\rho_{U}(\xi)}$ of any eigenfunction of $\mathcal{L}_{U}$ associated with eigenvalues with nonnegative real parts does not remove the eigenfunctions from $L^{2}(\mathbb{R})$. Therefore, since (2.31) does not possess eigenvalues with nonnegative real parts, with the exception of $\lambda=0$, then we conclude that $\mathcal{L}_{U}$ does not introduce unstable point spectrum in $\sigma_{p t}(\mathcal{L})$.

Next we take $U=0$ in (2.3) or equivalently in (2.9) and study the eigenvalue problem

$$
\mathcal{L}_{V} V=\lambda r_{V}(\xi) V .
$$

When $\lambda=0, V_{0}(\xi)=h^{\prime}(\xi)$ solves (2.33). It is important to notice that since $h(\xi)$ is monotone, the eigenfunction $h^{\prime}(\xi)$ associated with $\lambda=0$ does not have any zeros. Note that the following limits are finite:

$$
\begin{aligned}
& r_{V}^{+}=\lim _{\xi \rightarrow+\infty} r_{V}(\xi)=\lim _{\xi \rightarrow \infty} \frac{3}{\beta h(\xi)^{3}}=\frac{3}{\beta h_{R}^{3}}, \\
& r_{V}^{-}=\lim _{\xi \rightarrow-\infty} r_{V}(\xi)=\lim _{\xi \rightarrow-\infty} \frac{3}{\beta h(\xi)^{3}}=\frac{3}{\beta h_{L}^{3}}, \\
& p_{V}^{+}=\lim _{\xi \rightarrow+\infty} p_{V}(\xi)=\frac{h_{L} h_{R}+h_{L}^{2}-2 h_{R}^{2}}{\beta h_{R}^{3}}, \\
& p_{V}^{-}=\lim _{\xi \rightarrow-\infty} p_{V}(\xi)=\frac{h_{L} h_{R}+h_{R}^{2}-2 h_{L}^{2}}{\beta h_{L}^{3}} .
\end{aligned}
$$


Since

$$
\begin{aligned}
\exp \left(\int_{0}^{\xi} p_{V}(\tau) d \tau\right) & =\exp \left(\int_{\xi_{U}^{*}}^{\xi} 3 \frac{h^{3}(\tau)-3 s h(\tau)-3 K_{1}-\left(2 s h(\tau)+3 K_{1}\right)}{\beta h^{4}(\tau)} d \tau\right) \\
& =\exp \left(\int_{h_{*}}^{h(\xi)} 3 \frac{u^{3}-3 s u-3 K_{1}-\left(2 s h+3 K_{1}\right)}{u\left(u^{3}-3 s u-3 K_{1}\right)} d u\right) \\
& =K_{V}^{*}\left(\frac{h^{6}}{h+h_{L}+h_{R}}\right)\left(h-h_{R}\right)^{-1}\left(h-h_{L}\right)^{-1},
\end{aligned}
$$

from (2.27) and (2.28) it follows that the limits

$$
\rho_{V}^{ \pm}=\lim _{\xi \rightarrow \pm \infty} e^{-p_{V}^{ \pm} \xi} \rho_{V}(\xi)=\lim _{\xi \rightarrow \pm \infty} e^{-p_{V}^{ \pm} \xi}\left(r_{V}(\xi)\right)^{\frac{1}{2}} \exp \left(\int_{0}^{\xi} p_{V}(\tau) d \tau\right)
$$

are also finite and

$$
\lim _{\xi \rightarrow \pm \infty} q_{V}(\xi)=0 .
$$

Therefore, when we use Liouville-Green transformation (2.12), the multiplication of any eigenfunction that corresponds to any eigenvalue with positive real part with $\sqrt{\rho_{V}(\xi)}$ does not remove the product from $L^{2}(\mathbb{R})$. Moreover, $\lambda=0$ is an eigenvalue for the eigenvalue problem

$$
\mathcal{L}_{V}^{\rho} \eta_{V}=\lambda \eta_{V}
$$

with the eigenfunction $\eta_{s}^{0}(\xi)=\sqrt{\rho_{V}(\xi)} h^{\prime}(\xi)$ which has no zeros in $\mathbb{R}$. It follows then from [25, Theorem 2.3.3] that the self-adjoint operator $\mathcal{L}_{\eta}$ does not have positive eigenvalues, therefore, $\mathcal{L}_{V}$ does not have eigenvalues with positive real part.

Based on the analysis of operators $\mathcal{L}_{U}$ and $\mathcal{L}_{V}$, we conclude that the operator $\mathcal{L}$ in (2.9) does not have eigenvalues within the closed right half-plane with the exception of eigenvalues at zero. The operator $\mathcal{L}$ has at least a double eigenvalue at zero.

We next study the essential spectrum of the wavefront. In order to determine the Fredholm borders [25] of of the essential spectrum we consider the following limiting operators

$$
\mathcal{L}^{ \pm}=\lim _{\xi \rightarrow \pm \infty} \mathcal{L}=\left(\begin{array}{cc}
\frac{1}{r_{V}^{ \pm}}\left(\frac{d^{2}}{d \xi^{2}}+p_{V}^{ \pm} \frac{d}{d \xi}\right) & a_{V}^{ \pm} \frac{d^{2}}{d \xi^{2}} \\
0 & \frac{1}{r_{U}^{ \pm}}\left(\frac{\mathrm{d}^{2}}{\mathrm{~d} \xi^{2}}+p_{U}^{ \pm} \frac{\mathrm{d}}{\mathrm{d} \xi}\right)
\end{array}\right) .
$$

The curves produced by solving the determinant equation

$$
\operatorname{det}\left(\begin{array}{cc}
\frac{1}{r_{V}^{ \pm}}\left(-\kappa^{2}+i p_{V}^{ \pm} \kappa\right)-\lambda & -a_{V}^{ \pm} \kappa^{2} \\
0 & \frac{1}{r_{U}^{ \pm}}\left(-\kappa^{2}+i p_{U}^{ \pm} \kappa\right)-\lambda
\end{array}\right)=0,
$$

belong to the essential spectrum and form its boundaries. We recall that $\frac{1}{r_{U}^{ \pm}}=D$ and obtain the Fredholm borders of the essential spectrum

$$
\begin{aligned}
& \lambda_{1}^{ \pm}(\kappa)=D\left(-\kappa^{2}+i p_{U}^{ \pm} \kappa\right), \\
& \lambda_{2}^{ \pm}(\kappa)=\frac{1}{r_{V}^{ \pm}}\left(-\kappa^{2}+i p_{V}^{ \pm} \kappa\right),
\end{aligned}
$$

parametrized by $\kappa \in \mathbb{R}$. It is easy to see that the essential spectrum is confined to the closed left half plane. When $D \neq 0$ the essential spectrum touches the imaginary axes at the origin only (when $\kappa=0$ ). The spectrum of the linearization about the wavefront is schematically illustrated at Fig. 2.1. 


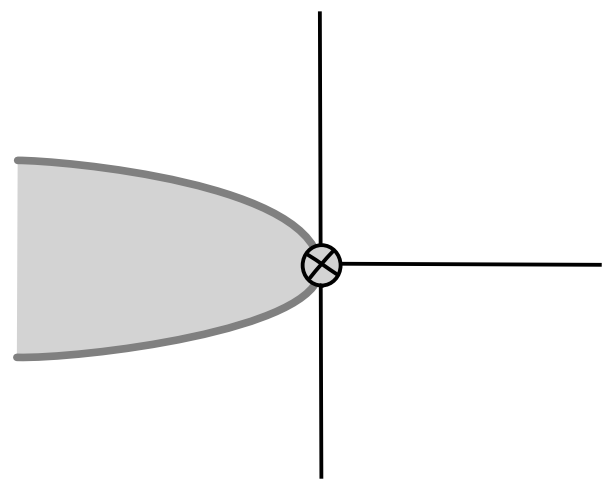

Figure 2.1. The location of the spectrum of the traveling wave with $\Gamma=0$. The continuous spectrum is bounded from the right by a parabola that touches the imaginary axes at $(0,0)$. There is a double eigenvalue at the origin.

\section{Spectrum in the CASe Where $\Gamma>0$}

In this and the next sections we study the spectrum of the linearization of the pde system about a wavefront with $\Gamma>0$.

The essential spectrum is obtained as in the previous section by computing the limiting operators (2.38). Since this procedure involves setting $\Gamma=0$, the essential spectrum in the case $\Gamma>0$ is the same as the one obtained for the case $\Gamma=0$. Furthermore, like in the case $\Gamma=0, \lambda=0$ is an eigenvalue of geometric multiplicity two. To prove this fact, one looks at the system (1.12) for $\lambda=0$. This system can be integrated once and the constant of integration can be set to zero as we are interested in the point spectrum on $L^{2}(\mathbb{R})$. To prove that the integrated system has two bounded solutions, one looks at the limiting systems (as $\xi \rightarrow \pm \infty)$. Then it is an easy exercise to solve those two constant systems and prove that, under condition (1.10), all the solutions of limiting system at $\infty$ (resp. $-\infty$ ) go to zero as $\xi \rightarrow \infty$ (resp. as $\xi \rightarrow-\infty)$. As a consequence, System (1.12) has two linearly independent solutions in $L^{2}(\mathbb{R})$.

We are now interested in finding a bound on any possible eigenvalue located on the right side of the complex plane. We assume that $\lambda$ is an eigenvalue of the of the problem $(1.12)$ with $\operatorname{Re}(\lambda)>0$. We want to find a bound on the modulus of $\lambda$. We prove the following lemma.

Lemma 3.1. Assume that $D, \beta, h, \Gamma$ are such that the nonlinear system of inequalities

$$
\begin{aligned}
& -\frac{4 \beta \min (h)^{3}}{3}+\max (h)^{2}(2+\beta \max (\Gamma)) r_{1}+\beta \max \left(h^{2} \Gamma\right) r_{2}<0, \\
& -4 D+\frac{\max (h)^{2}(2+\beta \max (\Gamma))}{r_{1}}+\frac{\beta \max \left(h^{2} \Gamma\right)}{r_{2}}<0
\end{aligned}
$$

has a positive solution $\left(r_{1}, r_{2}\right)$. If $\left(r_{3}, r_{4}, r_{5}\right)$ is chosen to satisfy

$$
\begin{aligned}
& \frac{\max (|q|)}{r_{3}}+\frac{\max (|g|) r_{5}}{2}=D-\frac{\max (h)^{2}(2+\beta \max (\Gamma))}{4 r_{1}}-\frac{\max \left(h^{2} \Gamma\right) \beta}{4 r_{2}}, \\
& r_{4}=\left(\frac{\min (h)^{3} \beta}{3}-\frac{r_{1} \max (h)^{2}(2+\beta \max (\Gamma))}{4}-\frac{\beta \max \left(h^{2} \Gamma\right) r_{2}}{4}\right) \frac{2}{\max (|f|)},
\end{aligned}
$$

then for the eigenvalues of the problem (1.12) with $\operatorname{Re}(\lambda)>0$ the following bound holds

$$
\operatorname{Re}(\lambda)+|\operatorname{Im}(\lambda)| \leq \frac{1}{2} \max \left[\max \left(f_{\xi}+2|q| r_{3}+\frac{|f|}{r_{4}}\right), \max \left(g_{\xi}+\frac{|g|}{r_{5}}\right)\right],
$$

where $f, g$, and $q$ are as defined in (3.6), (3.10) and (3.11), respectively. 
Proof. Since $\lambda$ is an eigenvalue $\lim _{\xi \rightarrow \pm \infty} U(\xi)=\lim _{\xi \rightarrow \pm \infty} V(\xi)=0$. This allows us to effectively use integration by parts formula as, for example, below.

We multiply the first equation of (1.12) by $\bar{V}$, integrate with respect to $\xi \in \mathbb{R}$ and, on the right hand side, do integration by parts once:

$$
\begin{aligned}
\lambda \int_{\mathbb{R}} V \bar{V} d \xi & =-\int_{\mathbb{R}}\left(s V+\frac{1}{2}\left(2 h \Gamma_{\xi} V+h^{2} U_{\xi}\right)-h^{2} V+\frac{\beta}{3}\left(3 h^{2} h_{\xi} V+h^{3} V_{\xi}\right)\right) \bar{V}_{\xi} d \xi \\
& =-\int_{\mathbb{R}}\left(\left(s+h \Gamma_{\xi}-h^{2}+\beta h^{2} h_{\xi}\right) V \bar{V}_{\xi}+\frac{h^{2}}{2} U_{\xi} \bar{V}_{\xi}+\frac{\beta h^{3}}{3}\left|V_{\xi}\right|^{2}\right) d \xi
\end{aligned}
$$

Next we notice that if $f$ is a real-valued function, then by integration by parts,

$$
\int_{\mathbb{R}} f V \bar{V}_{\xi} d \xi=-\int_{\mathbb{R}} \bar{V}(f V)_{\xi} d \xi=-\int_{\mathbb{R}} \bar{V}\left(f_{\xi} V+f V_{\xi}\right) d \xi=-\int_{\mathbb{R}}\left(f_{\xi}|V|^{2}+f \bar{V} V_{\xi}\right) d \xi,
$$

therefore

$$
2 \operatorname{Re}\left(\int_{\mathbb{R}} f V \bar{V}_{\xi} d \xi\right)=\int_{\mathbb{R}} f V \bar{V}_{\xi} d \xi+\int_{\mathbb{R}} f \bar{V} V_{\xi} d \xi=-\int_{\mathbb{R}} f_{\xi}|V|^{2} d \xi
$$

We use this equality for the first term in (3.4) with

$$
f=s+h \Gamma_{\xi}-h^{2}+\beta h^{2} h_{\xi} .
$$

Therefore,

$$
\operatorname{Re}(\lambda) \int_{\mathbb{R}}|V|^{2}=\frac{1}{2} \int_{\mathbb{R}} f_{\xi}|V|^{2} d \xi-\frac{1}{2} \operatorname{Re}\left(\int_{\mathbb{R}} h^{2} U_{\xi} \bar{V}_{\xi} d \xi\right)-\frac{\beta}{3} \int_{\mathbb{R}} h^{3}\left|V_{\xi}\right|^{2} d \xi .
$$

Next, we multiply the second equation of (1.12) by $\bar{U}$, integrate with respect to $\xi \in \mathbb{R}$ and, on the right hand side, do integration by parts once:

$\left.\lambda \int_{\mathbb{R}}|U|^{2}=-\int_{\mathbb{R}}\left[\left(s+h \Gamma_{\xi}-\frac{h^{2}}{2}\left(1-\beta h_{\xi}\right)\right) U \bar{U}_{\xi}+\Gamma\left(\Gamma_{\xi}-h+\beta h h_{\xi}\right)\right) V \bar{U}_{\xi}+\frac{\beta h^{2} \Gamma}{2} V_{\xi} \bar{U}_{\xi}+(D+h \Gamma)\left|U_{\xi}\right|^{2}\right] d \xi$.

The first term is treated similarly to (3.5), so

$$
\operatorname{Re}(\lambda) \int_{\mathbb{R}}|U|^{2} d \xi=\frac{1}{2} \int_{\mathbb{R}} g_{\xi}|U|^{2} d \xi-\operatorname{Re}\left(\int_{\mathbb{R}} q V \bar{U}_{\xi} d \xi\right)-\operatorname{Re}\left(\int_{\mathbb{R}} \frac{\beta h^{2} \Gamma}{2} V_{\xi} \bar{U}_{\xi} d \xi\right)-\int_{\mathbb{R}}(D+h \Gamma)\left|U_{\xi}\right|^{2} d \xi,
$$

where

$$
\begin{aligned}
g & =s+h \Gamma_{\xi}-\frac{1}{2} h^{2}+\frac{\beta}{2} h^{2} h_{\xi}, \\
q & =\Gamma\left(\Gamma_{\xi}-h+\beta h h_{\xi}\right) .
\end{aligned}
$$

We add the equation (3.9) and (3.7), and obtain

$$
\begin{aligned}
\operatorname{Re}(\lambda) \int_{\mathbb{R}}\left(|V|^{2}+|U|^{2}\right) d \xi= & \frac{1}{2} \int_{\mathbb{R}} f_{\xi}|V|^{2} d \xi+\frac{1}{2} \int_{\mathbb{R}} g_{\xi}|U|^{2} d \xi-\frac{\beta}{3} \int_{\mathbb{R}} h^{3}\left|V_{\xi}\right|^{2} d \xi-\int_{\mathbb{R}}(D+h \Gamma)\left|U_{\xi}\right|^{2} d \xi \\
& -\operatorname{Re}\left(\int_{\mathbb{R}} \frac{h^{2}}{2} U_{\xi} \bar{V}_{\xi} d \xi\right)-\operatorname{Re}\left(\int_{\mathbb{R}} \frac{\beta h^{2} \Gamma}{2} V_{\xi} \bar{U}_{\xi} d \xi\right)-\operatorname{Re}\left(\int_{\mathbb{R}} q V \bar{U}_{\xi} d \xi\right) .
\end{aligned}
$$

For the mixed terms we use Young's inequality $a b \leq \frac{a^{2}}{2 r}+\frac{r b^{2}}{2}$, where $r$ will be chosen later and $h \geq 0$

$$
\begin{aligned}
\left|\int_{\mathbb{R}} \frac{h^{2}}{2} U_{\xi} \bar{V}_{\xi} d \xi\right| & \leq \int_{\mathbb{R}} \frac{h^{2}}{2}\left(\frac{\left|U_{\xi}\right|^{2}}{2 r_{1}}+\frac{r_{1}\left|V_{\xi}\right|^{2}}{2}\right) d \xi, \\
\left|\int_{\mathbb{R}} \frac{h^{2} \Gamma \beta}{2} V_{\xi} \bar{U}_{\xi} d \xi\right| & \leq \int_{\mathbb{R}} \frac{\beta h^{2} \Gamma}{2}\left(\frac{\left|U_{\xi}\right|^{2}}{2 r_{2}}+\frac{r_{2}\left|V_{\xi}\right|^{2}}{2}\right) d \xi, \\
\left|\int_{\mathbb{R}} q V \bar{U}_{\xi} d \xi\right| & \leq \int_{\mathbb{R}}|q|\left(\frac{\left|U_{\xi}\right|^{2}}{2 r_{3}}+\frac{r_{3}|V|^{2}}{2}\right) d \xi .
\end{aligned}
$$


We use these inequalities in (3.12) to get

$$
\begin{aligned}
& \operatorname{Re}(\lambda) \int_{\mathbb{R}}\left(|V|^{2}+|U|^{2}\right) d \xi \leq \frac{1}{2} \int_{\mathbb{R}} f_{\xi}|V|^{2} d \xi+\frac{1}{2} \int_{\mathbb{R}} g_{\xi}|U|^{2} d \xi-\int_{\mathbb{R}} \frac{\beta h^{3}}{3}\left|V_{\xi}\right|^{2} d \xi-\int_{\mathbb{R}}(D+h \Gamma)\left|U_{\xi}\right|^{2} d \xi \\
& \quad+\int_{\mathbb{R}} \frac{h^{2}}{2}\left(\frac{\left|U_{\xi}\right|^{2}}{2 r_{1}}+\frac{r_{1}\left|V_{\xi}\right|^{2}}{2}\right) d \xi+\int_{\mathbb{R}} \frac{\beta h^{2} \Gamma}{2}\left(\frac{\left|U_{\xi}\right|^{2}}{2 r_{2}}+\frac{r_{2}\left|V_{\xi}\right|^{2}}{2}\right) d \xi+\int_{\mathbb{R}}|q|\left(\frac{\left|U_{\xi}\right|^{2}}{2 r_{3}}+\frac{r_{3}|V|^{2}}{2}\right) d \xi
\end{aligned}
$$

or

$$
\begin{aligned}
& \operatorname{Re}(\lambda) \int_{\mathbb{R}}\left(|V|^{2}+|U|^{2}\right) d \xi \leq \frac{1}{2} \int_{\mathbb{R}}\left(f_{\xi}+|q| r_{3}\right)|V|^{2} d \xi+\frac{1}{2} \int_{\mathbb{R}} g_{\xi}|U|^{2} d \xi \\
& \quad+\int_{\mathbb{R}}\left(\frac{r_{1}}{4}+\frac{\beta \Gamma r_{2}}{4}-\frac{h \beta}{3}\right) h^{2}\left|V_{\xi}\right|^{2} d \xi+\int_{\mathbb{R}}\left(-D-h \Gamma+\frac{h^{2}}{4 r_{1}}+\frac{h^{2} \Gamma \beta}{4 r_{2}}+\frac{|q|}{2 r_{3}}\right)\left|U_{\xi}\right|^{2} d \xi
\end{aligned}
$$

We introduce the following bounds:

$$
-\frac{\beta h^{3}}{3}+\frac{h^{2} r_{1}}{4}+\frac{\beta h^{2} \Gamma r_{2}}{4}=\frac{h^{2}}{4}\left(-\frac{4 \beta h}{3}+r_{1}+r_{2} \beta \Gamma\right) \leq \frac{h^{2}}{4}\left(-\frac{4 \beta \min (h)}{3}+r_{1}+r_{2} \beta \max (\Gamma)\right) .
$$

For the estimate on $\operatorname{Im}(\lambda)$, we use Young's inequality for the terms with $V \bar{V}_{\xi}$ and $U \bar{U}_{\xi}$,

$$
\begin{aligned}
\left|\int_{\mathbb{R}} f V \bar{V}_{\xi} d \xi\right| & \leq \int_{\mathbb{R}}|f|\left(\frac{|V|^{2}}{2 r_{4}}+\frac{r_{4}\left|V_{\xi}\right|^{2}}{2}\right) d \xi, \\
\left|\int_{\mathbb{R}} g U \bar{U}_{\xi} d \xi\right| & \leq \int_{\mathbb{R}}|g|\left(\frac{|U|^{2}}{2 r_{5}}+\frac{r_{5}\left|U_{\xi}\right|^{2}}{2}\right) d \xi .
\end{aligned}
$$

Taking imaginary part of (3.4) we get

$$
\operatorname{Im}(\lambda) \int_{\mathbb{R}}|V|^{2} d \xi=-\operatorname{Im}\left(\int_{\mathbb{R}}\left(f V \bar{V}_{\xi} d \xi+\frac{h^{2}}{2} U_{\xi} \bar{V}_{\xi}\right) d \xi\right)
$$

and taking imaginary part of (3.8) we get

$$
\operatorname{Im}(\lambda) \int_{\mathbb{R}}|U|^{2}=-\operatorname{Im}\left(\int_{\mathbb{R}}\left(g U \bar{U}_{\xi}+q V \bar{U}_{\xi}+\frac{\beta h^{2} \Gamma}{2} V_{\xi} \bar{U}_{\xi}\right) d \xi\right) .
$$

Adding (3.17) and (3.18) together and applying (3.13) and (3.16) we obtain

$$
\begin{aligned}
|\operatorname{Im}(\lambda)| \int_{\mathbb{R}}\left(|V|^{2}+|U|^{2}\right) d \xi & \leq\left|\int_{\mathbb{R}} f V \bar{V}_{\xi} d \xi\right|+\left|\int_{\mathbb{R}} g U \bar{U}_{\xi} d \xi\right|+\left|\int_{\mathbb{R}} q V \bar{U}_{\xi} d \xi\right|+\int_{\mathbb{R}} \frac{h^{2}(1+\beta \Gamma)}{2}\left|U_{\xi}\right|\left|V_{\xi}\right| d \xi \\
& \leq \int_{\mathbb{R}}|f|\left(\frac{|V|^{2}}{2 r_{4}}+\frac{r_{4}\left|V_{\xi}\right|^{2}}{2}\right) d \xi+\int_{\mathbb{R}} \frac{h^{2}(1+\beta \Gamma)}{2}\left(\frac{\left|U_{\xi}\right|^{2}}{2 r_{1}}+\frac{r_{1}\left|V_{\xi}\right|^{2}}{2}\right) d \xi \\
& +\int_{\mathbb{R}}|g|\left(\frac{|U|^{2}}{2 r_{5}}+\frac{r_{5}\left|U_{\xi}\right|^{2}}{2}\right) d \xi+\int_{\mathbb{R}}|q|\left(\frac{\left|U_{\xi}\right|^{2}}{2 r_{3}}+\frac{r_{3}|V|^{2}}{2}\right) d \xi,
\end{aligned}
$$

which simplifies to

$$
\begin{aligned}
|\operatorname{Im}(\lambda)| \int_{\mathbb{R}}\left(|V|^{2}+|U|^{2}\right) d \xi & \leq \int_{\mathbb{R}}\left(\frac{|f|}{2 r_{4}}+\frac{r_{3}|q|}{2}\right)|V|^{2} d \xi+\int_{\mathbb{R}}\left(\frac{r_{4}|f|}{2}+\frac{r_{1} h^{2}(1+\beta \Gamma)}{4}\right)\left|V_{\xi}\right|^{2} d \xi \\
& +\int_{\mathbb{R}}\left(\frac{h^{2}(1+\beta \Gamma)}{4 r_{1}}+\frac{r_{5}|g|}{2}+\frac{|q|}{2 r_{3}}\right)\left|U_{\xi}\right|^{2}+\int_{\mathbb{R}} \frac{|g|}{2 r_{5}}|U|^{2} d \xi .
\end{aligned}
$$


Adding (3.19) and (3.15) gives

$$
\begin{aligned}
(\operatorname{Re}(\lambda)+|\operatorname{Im}(\lambda)|) \int_{\mathbb{R}}\left(|V|^{2}+|U|^{2}\right) d \xi & \leq \int_{\mathbb{R}}\left(\frac{f_{\xi}}{2}+\frac{r_{3}|q|}{2}+\frac{|f|}{2 r_{4}}+\frac{r_{3}|q|}{2}\right)|V|^{2} d \xi+\int_{\mathbb{R}}\left(\frac{g_{\xi}}{2}+\frac{|g|}{2 r_{5}}\right)|U|^{2} d \xi \\
& +\int_{\mathbb{R}}\left(-D-h \Gamma+\frac{h^{2}}{4 r_{1}}+\frac{\beta h^{2} \Gamma}{4 r_{2}}+\frac{|q|}{r_{3}}+\frac{h^{2}(1+\beta \Gamma)}{4 r_{1}}+\frac{r_{5}|g|}{2}\right)\left|U_{\xi}\right|^{2} d \xi \\
& +\int_{\mathbb{R}}\left(-\frac{h^{3} \beta}{3}+\frac{r_{1} h^{2}}{4}+\frac{\beta h^{2} \Gamma r_{2}}{4}+\frac{r_{4}|f|}{2}+\frac{r_{1} h^{2}(1+\beta \Gamma)}{4}\right)\left|V_{\xi}\right|^{2} d \xi \\
& \leq \int_{\mathbb{R}}\left(\frac{f_{\xi}}{2}+\frac{|f|}{2 r_{4}}+r_{3}|q|\right)|V|^{2} d \xi+\int\left(\frac{g_{\xi}}{2}+\frac{|g|}{2 r_{5}}\right)|U|^{2} d \xi \\
& +\int_{\mathbb{R}}\left(-D-h \Gamma+\frac{h^{2}(2+\beta \Gamma)}{4 r_{1}}+\frac{\beta h^{2} \Gamma}{4 r_{2}}+\frac{|q|}{r_{3}}+\frac{r_{5}|g|}{2}\right)\left|U_{\xi}\right|^{2} d \xi \\
& +\int_{\mathbb{R}}\left(-\frac{h^{3} \beta}{3}+\frac{r_{1} h^{2}(2+\beta \Gamma)}{4}+\frac{\beta h^{2} \Gamma r_{2}}{4}+\frac{r_{4}|f|}{2}\right)\left|V_{\xi}\right|^{2} d \xi .
\end{aligned}
$$

We plan to use bounds

$$
\begin{aligned}
& -\frac{h^{3} \beta}{3}+\frac{r_{1} h^{2}(2+\beta \Gamma)}{4}+\frac{r_{2} \beta h^{2} \Gamma}{4}+\frac{r_{4}|f|}{2} \leq-\frac{\min (h)^{3} \beta}{3}+\frac{r_{1} \max (h)^{2}(2+\beta \max (\Gamma))}{4}+\frac{r_{2} \beta \max \left(h^{2} \Gamma\right)}{4}+\frac{r_{4} \max (|f|)}{2}, \\
& -D-h \Gamma+\frac{h^{2}(2+\beta \Gamma)}{4 r_{1}}+\frac{\beta h^{2} \Gamma}{4 r_{2}}+\frac{|q|}{r_{3}}+\frac{|g| r_{5}}{2} \leq-D+\frac{h^{2}(2+\beta \max (\Gamma))}{4 r_{1}}+\frac{\beta \max \left(h^{2} \Gamma\right)}{4 r_{2}}+\frac{\max (|q|)}{r_{3}}+\frac{r_{5} \max (|g|)}{2} .
\end{aligned}
$$

We now want to find conditions for being able to choose positive $r_{1}, r_{2}, r_{3}, r_{4}$, and $r_{5}$ such that

$$
\begin{aligned}
& -\frac{\min (h)^{3} \beta}{3}+\frac{r_{1} \max (h)^{2}(2+\beta \max (\Gamma))}{4}+\frac{r_{2} \beta \max \left(h^{2} \Gamma\right)}{4}+\frac{r_{4} \max (|f|)}{2} \leq 0, \\
& -D+\frac{\max (h)^{2}(2+\beta \max (\Gamma))}{4 r_{1}}+\frac{\beta \max \left(h^{2} \Gamma\right)}{4 r_{2}}+\frac{\max (|q|)}{r_{3}}+\frac{r_{5} \max (|g|)}{2} \leq 0 .
\end{aligned}
$$

It is obvious that we can find $r_{3}, r_{4}$, and $r_{5}$ satisfying (3.21) if and only if $r_{1}$ and $r_{2}$ satisfy (3.1). If there are such positive $r_{1}$ and $r_{2}$, then it suffices to choose $r_{3}, r_{4}$, and $r_{5}$ satisfying (3.2) for (3.21) to hold. Once (3.21) holds, the inequality (3.20) implies

$$
(\operatorname{Re}(\lambda)+|\operatorname{Im}(\lambda)|) \int_{\mathbb{R}}\left(|U|^{2}+|V|^{2}\right) d \xi \leq \int_{\mathbb{R}}\left(\frac{f_{\xi}}{2}+\frac{|f|}{2 r_{4}}+r_{3}|q|\right)|V|^{2} d \xi+\int\left(\frac{g_{\xi}}{2}+\frac{|g|}{2 r_{5}}\right)|U|^{2} d \xi,
$$

which in turn implies (3.3).

There is an alternative way to find a bound. It is done using the integrated coordinates $[16,19,26,33]$. These are obtained by making the substitution $(U, V)=\left(\widetilde{U}_{\xi}, \widetilde{V}_{\xi}\right)$ and integrating the equation to obtain

$$
\begin{aligned}
& \lambda V=s V_{\xi}+\frac{1}{2}\left(2 h \Gamma_{\xi} V_{\xi}+h^{2} U_{\xi \xi}\right)-h^{2} V_{\xi}+\frac{\beta}{3}\left(3 h^{2} h_{\xi} V_{\xi}+h^{3} V_{\xi \xi}\right), \\
& \lambda U=s U_{\xi}+\Gamma \Gamma_{\xi} V_{\xi}+h \Gamma_{\xi} U_{\xi}+h \Gamma U_{\xi \xi}-h \Gamma V_{\xi}-\frac{1}{2} h^{2} U_{\xi}+\frac{\beta}{2}\left(2 h \Gamma h_{\xi} V_{\xi}+h^{2} h_{\xi} U_{\xi}+h^{2} \Gamma V_{\xi \xi}\right)+D U_{\xi \xi},
\end{aligned}
$$

where we have suppressed the use of the tildes above the variables. The spectra of the eigenvalue problem (3.22) written in the integrated coordinates and the original problem (1.12) coincide except possibly at the origin (see for example Lemma 2.2 of [19]). The proof relies on the fact that if we have a solution to (1.12) then its anti-derivative solves the integrated version (3.22). If a solution of $(1.12)$ is in $L^{2}(\mathbb{R})$ for a nonzero value of $\lambda$, then it is possible to show that its anti-derivative also converges to zero as $|\xi| \rightarrow \infty$ by integrating (1.12). At $\lambda=0$, in our case, one of the eigenfunction of the original linear system (1.12) is made out of the derivatives of the two components of the front solution. As a consequence, the corresponding solution of the integrated system does not converge to zero. In view of the fact that $\lambda=0$ is an eigenvalue of geometric multiplicity two for the system (1.12), the geometric multiplicity of the zero eigenvalue of the integrated 
system is thus at most one. In fact, we verify numerically (using the Evans function technique described in the next section) that the eigenvalue problem (3.22) does not have any point spectrum at the origin. Using the integrated coordinate formulation (3.22) of the linear system (1.12), we prove the following lemma.

Lemma 3.2. Assume that $D, \beta, h, \Gamma$ are such that the nonlinear system of inequalities

$$
\begin{aligned}
& -\frac{4 \beta \min (h)^{3}}{3}+\max (h)^{2}(2+\beta \max (\Gamma)) r_{1}+\beta \max \left(h^{2} \Gamma\right) r_{2}<0, \\
& -4 D+\frac{\max (h)^{2}(2+\beta \max (\Gamma))}{r_{1}}+\frac{\beta \max \left(h^{2} \Gamma\right)}{r_{2}}<0
\end{aligned}
$$

has a positive solution $\left(r_{1}, r_{2}\right)$. If $\left(r_{3}, r_{4}, r_{5}, r_{6}, r_{7}\right)$ is chosen to satisfy (3.21), then for the eigenvalues of the problem (1.12) with $\operatorname{Re}(\lambda)>0$ the following bound holds

$$
\operatorname{Re}(\lambda)+|\operatorname{Im}(\lambda)| \leq \frac{1}{2} \max \left[\max \left(r_{7}\left|h h_{\xi}\right|-\tilde{f}_{\xi}+\frac{|\tilde{f}|}{r_{4}}+\frac{\left|h h_{\xi}\right|}{r_{6}}\right), \max \left(2 r_{3}|\tilde{q}|-\tilde{g}_{\xi}+\frac{|\tilde{g}|}{r_{5}}\right)\right]
$$

where $\tilde{f}, \tilde{g}$, and $\tilde{q}$ are as defined in $(3.26)$.

Proof. Using a very similar line of arguments as used in the proof of Lemma 3.1, we use the system (3.22) to prove the following bound:

$$
\begin{aligned}
(\operatorname{Re}(\lambda)+|\operatorname{Im}(\lambda)|) \int_{\mathbb{R}}\left(|V|^{2}+|U|^{2}\right) d \xi & \leq \frac{1}{2} \int_{\mathbb{R}}\left(2 r_{3}|\tilde{q}|-\tilde{g}_{\xi}+\frac{|\tilde{g}|}{r_{5}}\right)|U|^{2} d \xi \\
& +\frac{1}{2} \int_{\mathbb{R}}\left(r_{7}\left|h h_{\xi}\right|-\tilde{f}_{\xi}+\frac{|\tilde{f}|}{r_{4}}+\frac{\left|h h_{\xi}\right|}{r_{6}}\right)|V|^{2} d \xi \\
& +\int_{\mathbb{R}}\left(-\frac{h^{3} \beta}{3}+\frac{r_{2} \beta h^{2} \Gamma}{4}+\frac{r_{4}|\tilde{f}|}{2}+\frac{r_{1} h^{2}(2+\beta \Gamma)}{4}+\frac{|\tilde{q}|}{r_{3}}\right)\left|V_{\xi}\right|^{2} d \xi \\
& +\int_{\mathbb{R}}\left(-D-h \Gamma+\frac{\beta h^{2} \Gamma}{4 r_{2}}+\frac{\left|h h_{\xi}\right|}{2 r_{7}}+\frac{h^{2}(2+\beta \Gamma)}{4 r_{1}}+\frac{r_{5}|\tilde{g}|}{2}+\frac{r_{6}\left|h h_{\xi}\right|}{2}\right)\left|U_{\xi}\right|^{2} d \xi
\end{aligned}
$$

where

We plan to use bounds

$$
\begin{aligned}
& \tilde{f}=s+h \Gamma_{\xi}-h^{2}, \\
& \tilde{g}=s-h_{\xi} \Gamma-\frac{1}{2} h^{2}+\frac{\beta}{2} h^{2} h_{\xi}, \\
& \tilde{q}=\Gamma \Gamma_{\xi}-h \Gamma-\frac{\beta h^{2} \Gamma_{\xi}}{2} .
\end{aligned}
$$

$$
\begin{aligned}
& -\frac{h^{3} \beta}{3}+\frac{r_{2} \beta h^{2} \Gamma}{4}+\frac{r_{4}|\tilde{f}|}{2}+\frac{r_{1} h^{2}(2+\beta \Gamma)}{4}+\frac{|\tilde{q}|}{r_{3}} \leq \\
& -\frac{\min (h)^{3} \beta}{3}+\frac{r_{1} \max (h)^{2}(2+\beta \max (\Gamma))}{4}+\frac{r_{2} \beta \max \left(h^{2} \Gamma\right)}{4}+\frac{r_{4} \max (|\tilde{f}|)}{2}+\frac{\max (|\tilde{q}|)}{r_{3}}, \\
& -D-h \Gamma+\frac{\beta h^{2} \Gamma}{4 r_{2}}+\frac{\left|h h_{\xi}\right|}{2 r_{7}}+\frac{h^{2}(2+\beta \Gamma)}{4 r_{1}}+\frac{r_{5}|\tilde{g}|}{2}+\frac{r_{6}\left|h h_{\xi}\right|}{2} \leq \\
& -D+\frac{h^{2}(2+\beta \max (\Gamma))}{4 r_{1}}+\frac{\beta \max \left(h^{2} \Gamma\right)}{4 r_{2}}+\frac{r_{5} \max (|\tilde{g}|)}{2}+\frac{\left(1+r_{6} r_{7}\right) \max \left(\left|h h_{\xi}\right|\right)}{2 r_{7}} .
\end{aligned}
$$

We now want to find conditions for being able to choose positive $r_{i}, i=1, \ldots 7$, such that

$$
\begin{aligned}
& -\frac{\min (h)^{3} \beta}{3}+\frac{r_{1} \max (h)^{2}(2+\beta \max (\Gamma))}{4}+\frac{r_{2} \beta \max \left(h^{2} \Gamma\right)}{4}+\frac{r_{4} \max (|\tilde{f}|)}{2}+\frac{\max (|\tilde{q}|)}{r_{3}} \leq 0 \\
& -D+\frac{\max (h)^{2}(2+\beta \max (\Gamma))}{4 r_{1}}+\frac{\beta \max \left(h^{2} \Gamma\right)}{4 r_{2}}+\frac{r_{5} \max (|\tilde{g}|)}{2}+\frac{\left(1+r_{6} r_{7}\right) \max \left(\left|h h_{\xi}\right|\right)}{2 r_{7}} \leq 0 .
\end{aligned}
$$


It is obvious that we can find $r_{3}, r_{4}, r_{5}, r_{6}$, and $r_{7}$ satisfying (3.27) if and only if $r_{1}$ and $r_{2}$ satisfy (3.23). Once (3.21) holds, the inequality (3.25) implies

$$
\begin{array}{r}
(\operatorname{Re}(\lambda)+|\operatorname{Im}(\lambda)|) \int_{\mathbb{R}}\left(|U|^{2}+|V|^{2}\right) d \xi \leq \frac{1}{2} \int_{\mathbb{R}}\left(r_{7}\left|h h_{\xi}\right|-\tilde{f}_{\xi}+\frac{|\tilde{f}|}{r_{4}}+\frac{\left|h h_{\xi}\right|}{r_{6}}\right)|V|^{2} d \xi \\
+\frac{1}{2} \int_{\mathbb{R}}\left(2 r_{3}|\tilde{q}|-\tilde{g}_{\xi}+\frac{|\tilde{g}|}{r_{5}}\right)|U|^{2} d \xi,
\end{array}
$$

which in turn implies (3.24).

\section{Numerical Computations}

In this section, we present our numerical results concerning the existence and the stability. The front solutions are obtained through the numerical integration of the dynamical system (1.9) and the stability analysis is tackled by a numerical computation of the Evans function.

To obtain the heteroclinic orbit we numerically integrate (1.9) using the MatLab program ODE45. For given values of the parameters $\beta$ and $D$, we fix the initial conditions to be $h=-3 K_{1} / s$, and $\Gamma$ equal to its chosen maximum value. The system (1.9) then is integrated forward and backward in $\xi$. Figure 4.1 shows the solution in the case where $h_{L}=4, h_{R}=1, \beta=1, D=0.1$, and $\operatorname{Max}(\Gamma)=2$.
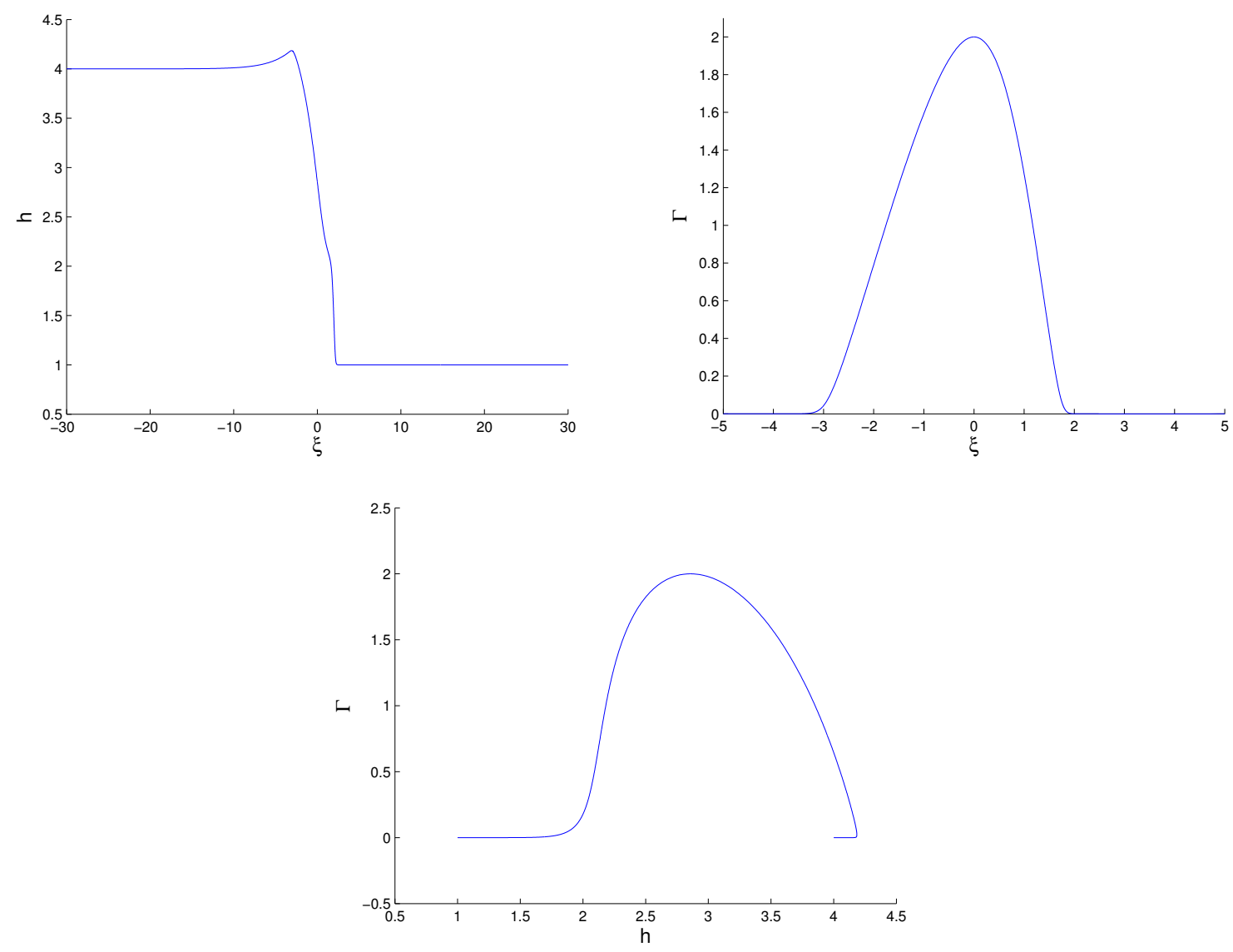

FiguRE 4.1. The heteroclinic orbit of system (1.9) in the case where $h_{L}=4, h_{R}=1, \beta=1$, $D=0.1$, and $\operatorname{Max}(\Gamma)=2$. The top figures show $h$ and $\Gamma$ as functions of $\xi$ and the bottom one shows the orbit in the $h-\Gamma$ plane. 
We are interested in locating the intersection of the open right half of the complex plane with the point spectrum of the eigenvalue problem $(1.12)$ on $L^{2}(\mathbb{R})$ using the Evans function. To do so, we use the integrated coordinates version of the linear system given in (3.22). As mentioned in the previous section, we verify numerically (using the Evans function technique described below) that the eigenvalue problem (3.22) does not have any point spectrum at the origin. This fact will simplify our computations as we will be able to perform our integrations on contours that include the origin.

We now write the eigenvalue problem (3.22) as a first order system

$$
\begin{aligned}
V_{1}^{\prime} & =\frac{12\left((s h+3 K 1) h^{2} \Gamma^{2}+\left(5 s h^{2} D+12 K 1 D h\right) \Gamma+12 K 1 D^{2}+8 s h D^{2}\right)}{\beta h^{4}(4 D+h \Gamma)^{2}} V_{1} \\
& +\frac{12 \lambda(h \Gamma+D)}{h^{3} \beta(4 D+h \Gamma)} V_{2}-\frac{12 D\left(s h+3 K_{1}\right)}{\beta h^{2}(h \Gamma+4 D)^{2}} U_{1}-\frac{6 \lambda}{(h \Gamma+4 D) h \beta} U_{2}, \\
V_{2}^{\prime} & =V_{1}, \\
U_{1}^{\prime} & =\frac{8 D\left(s h+3 K_{1}\right)}{h(h \Gamma+4 D)^{2}} U_{1}+\frac{4 \lambda}{h \Gamma+4 D} U_{2}-\frac{2 \Gamma\left(12 K_{1} D+s h^{2} \Gamma+6 K_{1} h \Gamma\right)}{h^{2}(h \Gamma+4 D)^{2}} V_{1}-\frac{6 \lambda \Gamma}{h(h \Gamma+4 D)} V_{2}, \\
U_{2}^{\prime} & =U_{1},
\end{aligned}
$$

where $h$ and $\Gamma$ solve (1.9). Then, (4.1) is a linear dynamical system of the form

$$
X^{\prime}=A(\xi, \lambda) X
$$

where $X=\left(V_{1}, V_{2}, U_{1}, U_{2}\right)$ and $A$ is the $4 \times 4$ following square matrix

$$
A(\xi, \lambda)=\left(\begin{array}{cccc}
\frac{12\left((s h+3 K 1) h^{2} \Gamma^{2}+\left(5 h^{2} D+12 K 1 D h\right) \Gamma+12 K 1 D^{2}+8 s h D^{2}\right)}{\beta h^{4}(4 D+h \Gamma)^{2}} & \frac{12 \lambda(h \Gamma+D)}{h^{3} \beta(4 D+h \Gamma)} & -\frac{12 D\left(s h+3 K_{1}\right)}{\beta h^{2}(h \Gamma+4 D)^{2}} & -\frac{6 \lambda}{(h \Gamma+4 D) h \beta} \\
1 & 0 & 0 & 0 \\
-\frac{2 \Gamma\left(12 K_{1} D+s h^{2} \Gamma+6 K_{1} h \Gamma\right)}{h^{2}(h \Gamma+4 D)^{2}} & -\frac{6 \lambda \Gamma}{h(h \Gamma+4 D)} & \frac{8 D\left(s h+3 K_{1}\right)}{h(h \Gamma+4 D)^{2}} & \frac{4 \lambda}{h \Gamma+4 D} \\
0 & 0 & 1
\end{array}\right) .
$$

The asymptotic behavior as $\xi \rightarrow \pm \infty$ of the solutions to (4.2) is determined by the matrices

$$
A^{ \pm \infty}(\lambda)=\lim _{\xi \rightarrow \pm \infty} A(\xi, \lambda)
$$

which are found by inserting the values $\Gamma=0$ and $h=h_{R}$ or $h_{L}$ in $A$ :

$$
\begin{gathered}
A^{\infty}(\lambda)=\left(\begin{array}{cccc}
\frac{2 h_{R}^{2}-h_{L}^{2}-h_{L} h_{R}}{\beta h_{R}^{3}} & \frac{3 \lambda}{h_{R}^{3} \beta} & \frac{2 h_{L}^{2}+2 h_{L} h_{R}-h_{R}^{2}}{4 \beta D h_{R}} & -\frac{3 \lambda}{2 \beta D h_{R}} \\
1 & 0 & 0 & 0 \\
0 & 0 & \frac{h_{R}^{2}-2 h_{L}^{2}-2 h_{L} h_{R}}{6 D} & \frac{\lambda}{D} \\
0 & 0 & 1 & 0
\end{array}\right), \\
A^{-\infty}(\lambda)=\left(\begin{array}{cccc}
\frac{2 h_{L}^{2}-h_{R}^{2}-h_{L} h_{R}}{\beta h_{L}^{3}} & \frac{3 \lambda}{h_{L}^{3} \beta} & \frac{2 h_{R}^{2}+2 h_{L} h_{R}-h_{L}^{2}}{4 \beta D h_{L}} & -\frac{3 \lambda}{2 \beta D h_{L}} \\
1 & 0 & 0 & 0 \\
0 & 0 & \frac{h_{L}^{2}-2 h_{R}^{2}-2 h_{L} h_{R}}{6 D} & \frac{\lambda}{D} \\
0 & 0 & 1 & 0
\end{array}\right) .
\end{gathered}
$$


For $\operatorname{Re}(\lambda)>0$, both these matrices have two eigenvalues with positive real parts and two with negative real parts. We denote the two eigenvalues of $A^{\infty}$ with negative real parts as $\mu_{i+}$ with eigenvectors $v_{i+}, \quad i=1,2$, and the two eigenvalues of $A^{-\infty}$ with positive real parts as $\mu_{i-}$ with eigenvectors $v_{i-}, \quad i=1,2$. The precise expressions for these are given in Appendix A. As a consequence, the system (4.2) has two linearly independent solutions $X_{1+}$ and $X_{2+}$ converging to zero as $\xi \rightarrow \infty$ and two solutions $X_{1-}$ and $X_{2-}$ converging to zero as $\xi \rightarrow-\infty$, satisfying

$$
\lim _{\xi \rightarrow \pm \infty} X_{i \pm} e^{-\mu_{i \pm} \xi}=v_{i \pm}, \quad i=1,2
$$

Clearly, $\lambda_{0}$ is an eigenvalue for the problem (4.1) if and only if space of solutions of (4.2) bounded as $\xi \rightarrow+\infty$, spanned by $\left\{X_{1+}, X_{2+}\right\}$, and the space of solutions bounded as $\xi \rightarrow-\infty$, spanned by $\left\{X_{1-}, X_{2-}\right\}$, have an intersection of strictly positive dimension when $\lambda=\lambda_{0}$. The most straightforward way to test whether these two spaces of solutions intersect non-trivially is to calculate the determinant of the two spanning sets, evaluated at some value of $\xi$ (usually taken as $\xi=0$ ). This function is called the Evans function $[2,12,15,22-24,31,35,36,39]$. It is analytic on to the right of the essential spectrum and it is real for $\lambda$ real. Numerically, the two solutions $X_{1+}$ and $X_{2+}$ are obtained by integrating (4.2) backwards from a sufficiently large positive value of $\xi$, with initial conditions in the $v_{1+}$ and $v_{2+}$, respectively. Even though these two vectors are linearly independent, the numerical integration will lead to an alignment with the eigendirection corresponding to the eigenvalue with smallest real part. To circumvent this problem, the Evans function is computed using an alternative definition involving exterior algebra $[1,3,5,7-9,13,34,37]$. We are in the situation where the dimension of the system is $n=4$ and the dimensions of the stable and unstable manifolds are $n_{s}=n_{U}=2$. In such a case, we consider the wedge-space $\bigwedge^{2}\left(\mathbb{C}^{4}\right)$, the space of all two forms on $\mathbb{C}^{4}$. The induced dynamics of $(4.2)$ on $\bigwedge^{2}\left(\mathbb{C}^{4}\right)$ can be written as

$$
U^{\prime}=\mathbf{A}^{(2)}(\xi, \lambda) U \text {. }
$$

Here the matrix $A^{(2)}$ is matrix generated by $A=\left\{a_{i j}\right\}$ on the wedge-space $\bigwedge^{2}\left(\mathbb{C}^{4}\right)$. Using the standard basis of $\bigwedge^{2}\left(\mathbb{C}^{4}\right)$,

$$
\begin{aligned}
& \omega_{1}=\mathbf{e}_{1} \wedge \mathbf{e}_{2}, \quad \omega_{2}=\mathbf{e}_{1} \wedge \mathbf{e}_{3}, \quad \omega_{3}=\mathbf{e}_{1} \wedge \mathbf{e}_{4}, \\
& \omega_{4}=\mathbf{e}_{2} \wedge \mathbf{e}_{3}, \quad \omega_{5}=\mathbf{e}_{2} \wedge \mathbf{e}_{4}, \quad \omega_{6}=\mathbf{e}_{3} \wedge \mathbf{e}_{4},
\end{aligned}
$$

where $\left\{\mathbf{e}_{1}, \mathbf{e}_{2}, \mathbf{e}_{3}, \mathbf{e}_{4}\right\}$ is the standard basis of $\mathbb{C}^{4}$, the matrix $A^{(2)}$ is given by

$$
A^{(2)}=\left(\begin{array}{cccccc}
a_{11}+a_{22} & a_{23} & a_{24} & -a_{13} & -a_{14} & 0 \\
a_{32} & a_{11}+a_{33} & a_{34} & a_{12} & 0 & -a_{14} \\
a_{42} & a_{43} & a_{11}+a_{44} & 0 & a_{12} & a_{13} \\
-a_{31} & a_{21} & 0 & a_{22}+a_{33} & a_{34} & -a_{24} \\
-a_{41} & 0 & a_{21} & a_{43} & a_{22}+a_{44} & a_{23} \\
0 & -a_{41} & a_{31} & -a_{42} & a_{32} & a_{33}+a_{44}
\end{array}\right) .
$$

The asymptotic matrices are then given by

$$
\lim _{\xi \rightarrow \pm \infty} A^{(2)}(\xi, \lambda)=\left(\mathcal{A}^{ \pm \infty}\right)^{(2)}
$$

where $\mathcal{A}^{ \pm \infty}$ are given in (4.4). The eigenvalue of $\left(\mathcal{A}^{\infty}\right)^{(2)}$ with smallest real part is given by $\mu_{1+}+\mu_{2+}$ with eigenvector $v_{1+} \wedge v_{2+}$. The solution of (4.5) given by $U_{+}=X_{1+} \wedge X_{2+}$ then behaves as

$$
\lim _{\xi \rightarrow \infty} U_{+} e^{-\left(\mu_{1+}+\mu_{1+}\right) \xi}=w_{+} \equiv v_{1+} \wedge v_{2+} .
$$

Similarly, the solution $U_{-}=X_{1-} \wedge X_{2-}$ behaves as

$$
\lim _{\xi \rightarrow-\infty} U_{-} e^{-\left(\mu_{1-}+\mu_{-}\right) \xi}=w_{-} \equiv v_{1-} \wedge v_{2-} .
$$


This allows us to define the Evans function as

$$
E(\lambda) \equiv U_{-} \wedge U_{+} \cdot
$$

If $U$ is written in components in the basis (4.6), then the Evans function is computed $[3,6,7]$ as

$$
E(\lambda)=U_{-}^{T} \Sigma U_{+},
$$

where $\Sigma$ is the matrix

$$
\boldsymbol{\Sigma}=\left[\begin{array}{rrrrrr}
0 & 0 & 0 & 0 & 0 & 1 \\
0 & 0 & 0 & 0 & -1 & 0 \\
0 & 0 & 0 & 1 & 0 & 0 \\
0 & 0 & 1 & 0 & 0 & 0 \\
0 & -1 & 0 & 0 & 0 & 0 \\
1 & 0 & 0 & 0 & 0 & 0
\end{array}\right]
$$

and where $U_{ \pm}$are evaluated at $\xi=0$.

A note about the region of analyticity of the Evans function in the complex plane. The function $E(\lambda)$ will be analytic in the any region of the complex plane where the eigenvalues $\mu_{1+}+\mu_{2+}$ and $\mu_{1-}+\mu_{2-}$ are, respectively, the eigenvalues with smallest and largest real part of $\left(\mathcal{A}^{\infty}\right)^{(2)}$ and $\left(\mathcal{A}^{-\infty}\right)^{(2)}$. In view of the expressions for the eigenvalues given in Appendix A, to define such a region, it suffices to implement the condition

$$
\operatorname{Re}(\lambda)>-\min \left(\frac{\left(2 h_{R}{ }^{2}-h_{L}{ }^{2}-h_{L} h_{R}\right)^{2}}{12 h_{R}{ }^{3} \beta}, \quad \frac{\left(h_{R}{ }^{2}-2 h_{L}{ }^{2}-2 h_{L} h_{R}\right)^{2}}{144 D}, \quad \frac{\left(2 h_{L}{ }^{2}-h_{R}{ }^{2}-h_{R} h_{L}\right)^{2}}{12 h_{L}{ }^{3} \beta}, \quad \frac{\left(h_{L}{ }^{2}-2 h_{R}{ }^{2}-2 h_{R} h_{L}\right)^{2}}{144 D}\right) .
$$

To compute the Evans numerically, we choose a positive value $\xi=L$ at which the matrix given $A$ is suitably close to its asymptotic value $\mathcal{A}^{\infty}$. We then integrate the system (4.5) backward from $\xi=L$ in the direction of the eigenvector $w_{+}$and find $U_{+}(0)$. Additionally, in order to eliminate the exponential growth due to the eigenvalue with negative real part as we integrate from $\xi=L$, we modify the system (4.5) in the following way

$$
U^{\prime}=\left(A^{(2)}-\left(\mu_{1+}+\mu_{2+}\right) I\right) U,
$$

and use the initial condition $X(L)=w_{+}$. Similarly, we find $U_{-}(0)$ by integrating the system (4.5) from $\xi=-L$ to 0 . Note that in the numerical computations, we choose the eigenvectors $w_{ \pm}$so that $w_{-}^{T} \Sigma w_{+}=1$. This choice has the convenient consequence that $\lim _{|\lambda| \rightarrow \infty} E(\lambda)=1$.

Since we are interested in the zeroes of the Evans function, the standard method is to compute the integral of the logarithmic derivative of the Evans function on a given closed curve and obtain the winding number of $E(\lambda)$ along that curve. In order to numerically verify that there are no zeroes of the Evans function inside a given region of the complex plane, we choose a closed curve whose points satisfy (4.9) and whose interior encloses the region.

For example, it can be verified numerically that the eigenvalue problem (3.22) has no eigenvalue at $\lambda=0$ by performing a winding number computation on a small closed curve surrounding the origin. Once this is done, we can choose a contour of integration which include the origin as a point. For example, in the case $h_{L}=4, h_{R}=1, D=0.1, \beta=1$, and $\max (\Gamma)=2$, we choose our closed curve to be the boundary of the right half of the circle of radius 5 and center at the origin. Our numerical winding number computation then shows that the Evans function has no zero inside that region as the winding number is found to be 0. Figure 4.2 shows the graph of the Evans function for values of $\lambda$ on the boundary of that right half circle. On the figure the winding number of the Evans function is shown to be zero as the curve wraps around the origin twice, once in each direction. 
While our search was not extensive, we have made similar computations for a few other values of the parameters $h_{L}, h_{R}, D, \beta$, and $\max (\Gamma)$. Each time, we have not found zeros of the Evans functions. Note that those computations only show the absence of eigenvalues in a certain domain and do not imply spectral stability since there is always the possibility of unstable eigenvalues outside a considered domain.

In order to numerically prove spectral stability, we will use the bounds provided by Lemma 3.1 or Lemma 3.2. In order to use the bound provided by Lemma 3.1, we need to choose a set of parameter values for which there exist positive $r_{1}$ and $r_{2}$ satisfying System (3.1) and whose bound given by (3.3) is of a reasonable size. Note that a necessary and sufficient condition for the System (3.1) to hold for some positive $r_{1}$ and $r_{2}$ can be found. To do so, it suffices to replace the inequalities in System (3.1) by equalities and check if the two curves defined by this new system intersect in the $\left(r_{1}, r_{2}\right)$ plane. The condition takes the form on an inequality involving the different parameters used in System (3.1). We choose not to write this inequality explicitly as it is rather cumbersome. Once we know that we can find positive $r_{1}$ and $r_{2}$ satisfying (3.1) we choose the quantities $r_{1}, r_{2}, r_{3}, r_{4}$, and $r_{5}$ by numerically finding the ones that make the bound provided by the RHS of (3.3) to be the smallest possible. Applying this strategy, we find that for $h_{L}=4, h_{R}=1$, $\beta=22, D=70$, and $\operatorname{Max}(\Gamma)=0.1$, the values $r_{1}=0.3211, r_{2}=0.3205, r_{3}=18.5206, r_{4}=0.0296$, and $r_{5}=0.0501$ satisfy (3.1) and (3.2). The bound provided by (3.3) is then computed to be 152.4339. In order to numerically verify that there are no zeroes of the Evans function on the right side of the complex plane, we choose a closed curve whose interior encloses the region defined by (3.3). In that case, it suffices to consider the right half of the circle centered at the origin with radius larger than the value of the bound. Our numerical winding number computation then shows that the Evans function has no other zero than the one at the origin. Note that we can reduce our numerical computations by using the fact that the Evans function satisfy the property that $D(\bar{\lambda})=\overline{D(\lambda)}$. Indeed, since we choose our contour of integration to be symmetric with respect to the imaginary axis, it is sufficient to perform our numerical integration along the upper (or lower) half of the half-circle to determine the winding number. Note that we have also used Lemma 3.2 for this particular set of parameters and have found a bound of 245.6973. Thus, in this case, we have used the better bound equal to 152.4339 provided by Lemma 3.1. Another set of values we have considered is $h_{L}=3, h_{R}=1, \beta=200, D=90$, and $\operatorname{Max}(\Gamma)=0.0822$. In this case, the radius provided by Lemma 3.1 is 1.3306 while the radius provided by Lemma 3.2 is 1.8203 . We were thus also able to numerically show spectral stability for those values of the parameters as well.

\section{Acknowledgements.}

The authors thank J. Humpherys for bringing their attention to the importance of the integrated variables. We appreciate the assistance of J. Mueller with Redhawk Cluster (Research Computing Support, Miami University).

We gratefully acknowledge the support of the National Science Foundation through grants DMS-1311313 (A. Ghazaryan) and DMS-0908074 (S. Lafortune) and of the Simons Foundation through the Collaboration grant \#246535 (V. Manukian).

Appendix A. Eigenvalues And eigenvectors for the matrices in (4.4)

The two eigenvalues with negative real part for $A^{\infty}(\lambda)$ when $\operatorname{Re}(\lambda)>0$ are

$$
\begin{gathered}
\mu_{1+}=\frac{2 h_{R}^{2}-h_{L}^{2}-h_{L} h_{R}-\sqrt{\left(2 h_{R}^{2}-h_{L}^{2}-h_{L} h_{R}\right)^{2}+12 h_{R}^{3} \beta \lambda}}{2 h_{R}^{3} \beta}, \\
\mu_{2+}=\frac{h_{R}^{2}-2 h_{L}^{2}-2 h_{L} h_{R}-\sqrt{\left(h_{R}^{2}-2 h_{L}^{2}-2 h_{L} h_{R}\right)^{2}+144 D \lambda}}{12 D},
\end{gathered}
$$




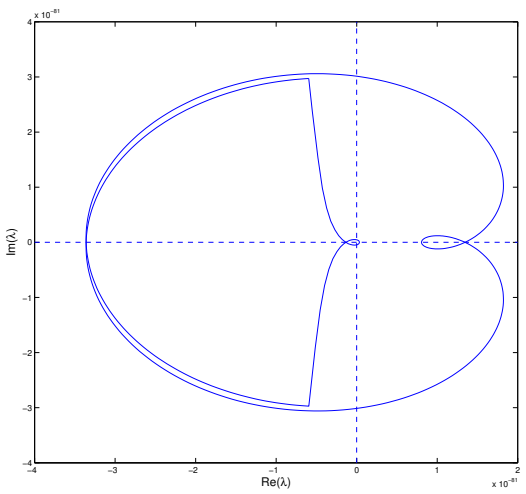

(a)

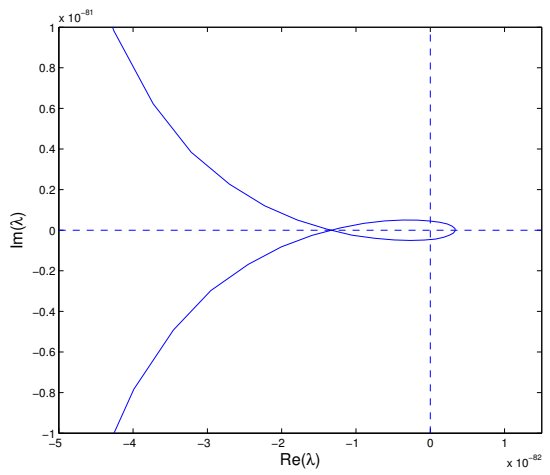

(b)

FiguRE 4.2. Figure showing the graph of the Evans function for values of $\lambda$ on the boundary of the right half of the circle of radius 5 and center at the origin (with $h_{L}=4, h_{R}=1$, $D=0.1, \beta=1$, and $\max (\Gamma)=2$ ). The plot (a) shows the whole graph while the plot (b) shows a close up around the origin. The winding number of the Evans function is zero as the curve wraps around the origin twice, once in each direction.

with eigenvectors

$$
\begin{aligned}
& v_{1+}=\left(0,0, \mu_{1+}, 1\right)^{T} \\
& v_{2+}=\left(\frac{\mu_{2+}\left(-D h_{R}^{2} \tilde{\mu}_{2+}+2 \lambda\left(D-h_{R}^{3} \beta / 3\right)\right)}{\lambda h_{R}^{2}}, \frac{-D h_{R}^{2} \tilde{\mu}_{2+}+2 \lambda\left(D-h_{R}^{3} \beta / 3\right)}{\lambda h_{R}^{2}}, \mu_{2+}, 1\right),
\end{aligned}
$$

where $\tilde{\mu}_{2+}$ is the conjugate of $\mu_{2+}$.

The two eigenvalues with positive real part for $A^{-\infty}(\lambda)$ when $\operatorname{Re}(\lambda)>0$ are

$$
\begin{gathered}
\mu_{1-}=\frac{2 h_{L}{ }^{2}-h_{R}^{2}-h_{R} h_{L}+\sqrt{\left(2 h_{L}^{2}-h_{R}^{2}-h_{R} h_{L}\right)^{2}+12 h_{L}^{3} \beta \lambda}}{2 h_{L}^{3} \beta}, \\
\mu_{2-}=\frac{h_{L}{ }^{2}-2 h_{R}^{2}-2 h_{R} h_{L}+\sqrt{\left(h_{L}^{2}-2 h_{R}^{2}-2 h_{R} h_{L}\right)^{2}+144 D \lambda}}{12 D},
\end{gathered}
$$

with eigenvectors

$$
\begin{aligned}
& v_{1-}=\left(0,0, \mu_{1-}, 1\right)^{T} \\
& v_{2-}=\left(\frac{\mu_{2-}\left(-D h_{L}^{2} \tilde{\mu}_{2-}+2 \lambda\left(D-h_{L}^{3} \beta / 3\right)\right)}{\lambda h_{L}^{2}}, \frac{-D h_{L}^{2} \tilde{\mu}_{2-}+2 \lambda\left(D-h_{L}^{3} \beta / 3\right)}{\lambda h_{L}^{2}}, \mu_{2-}, 1\right),
\end{aligned}
$$

where $\tilde{\mu}_{2-}$ is the conjugate of $\mu_{2-}$.

\section{REFERENCES}

[1] A. L. Afendikov and T. J. Bridges, Instability of the Hocking-Stewartson pulse and its implications for the threedimensional Poiseuille flow, R. Soc. Lond. Proc. Ser. A Math. Phys. Eng. Sci. 457 (2001) 257-272.

[2] J. Alexander, R. Gardner, and C. Jones, A topological invariant arising in the stability analysis of travelling waves, J. Reine Angew. Math. 410 (1990) 167-212.

[3] L. Allen and T. J. Bridges, Numerical exterior algebra and the compound matrix method, Numer. Math. 92 (2002) 197-232.

[4] R. ARIs, Vectors, Tensors, and the Basic Equations of Fluid Dynamics, PrenticeĐHall, Englewood Cliffs, NJ, 1962. 
[5] T. J. Bridges, The Orr-Sommerfeld equation on a manifold, R. Soc. Lond. Proc. Ser. A Math. Phys. Eng. Sci. 455 (1999) 3019-3040.

[6] T. J. Bridges and G. Derks, Hodge duality and the Evans function, Phys. Lett. A 251 (1999) 363-372.

[7] T. J. Bridges, G. Derks, and G. Gottwald, Stability and instability of solitary waves of the fifth-order KdV equation: A numerical framework, Phys. D 172 (2002) 190-216.

[8] L. Q. BRIN, Numerical testing of the stability of viscous shock waves, Math. Comp. 70 (2001) 1071-1088.

[9] G. Derks and G. A. Gottwald, A robust numerical method to study oscillatory instability of gap solitary waves, SIAM J. Appl. Dyn. Syst. 4 (2005) 140-158.

[10] B. D. Edmonstone, R. V. Craster, and O. K. Matar, Flow of surfactant-laden thin films down an inclined plane, J. Engrg. Math. 50 (2004) 141-156.

[11] B. D. Edmonstone, R. V. Craster, and O. K. Matar, Surfactant-induced fingering phenomena in thin film flow down an inclined plane, Phys. D 209 (2005) 62-79.

[12] J. W. Evans, Nerve axon equations. IV. The stable and unstable impulse, Indiana Univ. Math. J. 24 (1975) 1169-1190.

[13] V. Gubernov, G. N. Mercer, H. S. Sidhu, and R. O. Weber, Evans function stability of combustion waves, SIAM J. Appl. Math. 63 (2003) 1259-1275.

[14] B. J. Fischer And S. M. Troian, Thinning and disturbance growth in liquid films mobilized by continuous surfactant delivery, Phys. Fluids 15 (2003) 3837-3845.

[15] R. A. Gardner and K. Zumbrun, The Gap Lemma and geometric criteria for instability of viscous shock profiles, Communications on Pure and Applied Mathematics LI (1998) 0797-0855.

[16] J. Goodman, Nonlinear asymptotic stability of viscous shock profiles for conservation laws, Arch. Rational Mech. Anal.95 325-344 (1986).

[17] D. Halpern, J. L. Bull, and J. B. Grotberg, The effect of airway wall motion on surfactant delivery, J. Biomech. Eng. 126 (2004) 410-419.

[18] D. Halpern, O. E. Jensen, and J. B. Grotberg, A theoretical study of surfactant and liquid delivery into the lung, J. Appl. Physiol. 1 (1998) 333-352.

[19] J. Humpherys, Spectral energy methods and the stability of shock waves, Doctoral dissertation, Indiana University (2002).

[20] O. E. Jensen and J. B. Grotberg, Insoluble surfactant spreading on a thin viscous film: Shock evolution and film rupture, J. Fluid Mech. 240 (1992), pp. 259-288.

[21] O. E. Jensen And J. B. Grotberg, The spreading of heat or soluble surfactant along a thin liquid film, Phys. Fluids A, 5 (1993), 58-68.

[22] C. K. R. T. Jones, Stability of the travelling wave solution to the FitzHugh-Hagumo equation, Trans. AMS 286 (1984) 431-469.

[23] T. Kapitula And B. Sandstede, Stability of bright solitary-wave solutions to perturbed nonlinear Schrödinger equations, Physica D 124 (1998) 58-103.

[24] T. Kapitula And J. Rubin, Existence and stability of standing hole solutions to complex Ginzburg-Landau equations, Nonlinearity 13 (2000) 77-112.

[25] T. Kapitula and K. Promislow, Spectral and Dynamical Stability of Nonlinear Waves, Applied Mathematical Sciences 185, Springer, 2013.

[26] S. Kawashima And A. Matsumura, Asymptotic stability of traveling wave solutions of systems for one-dimensional gas motion, Comm. Math. Phys. 101 (1985) 97-127.

[27] O. K. Matar And S. M. Troian, Spreading of a surfactant monolayer on a thin liquid film: Onset and evolution of digitated structures, Chaos 9 (1999) 141-153.

[28] F. Olver, Asymptotics and Special Functions, Computer science and applied mathematics, Academic Pr; 1 st edition, 1974.

[29] R. Levy and M. Shearer, The motion of a thin liquid film driven by surfactant and gravity, SIAM J. Appl Math. 66 (2006) 1588-1609.

[30] R. Levy, M. Shearer, And T. Witelski, Gravity-driven thin liquid films with insoluble surfactant: smooth traveling waves, Eur. J. Appl. Math. 18 (2007) 679-708.

[31] Y.A. Li and K. Promislow, The mechanism of the polarizational mode instability in birefringent fiber optics, SIAM J. Math. Anal. 31 (2000), 1351-1373.

[32] V. Manukian and S. Schecter, Traveling waves for a thin liquid film with surfactant on an inclined plane, Nonlinearity 22 (2009) 85-122.

[33] A. Matsumura and K. Nishinara, On the stability of travelling wave solutions of a one-dimensional model system for compressible viscous gas, Japan J. Appl. Math. 2 (1985) 17-25. 
[34] B. NG And W. ReID, An initial-value method for eigenvalue problems using compound matrices, J. Comput. Phys. 30 (1979) 125-136.

[35] R. Pego and M. Weinstein, Eigenvalues, and instabilities of solitary waves, Phil. Trans. R. Soc. London A 340 (1992) 47-94.

[36] B. Sandstede, Stability of travelling waves, In: Handbook of Dynamical Systems II: Towards Applications, 983-1055, (B. Fiedler, ed.), Elsevier (2002).

[37] P. Simon, S. Kalliadasis, J. Merkin, And S. Scott, Evans function analysis of the stability of non-adiabatic flames, Combust. Theory Model. 7 (2003) 545-561.

[38] H. A. Stone, A simple derivation of the time-dependent convective-diffusion equation for surfactant transport along a deforming interface, Phys. Fluids A, 2 (1990), 111-112.

[39] E. YanaGidA, Stability of fast traveling pulse solutions of the FitzHugh-Nagumo equations, J. Math. Biol. 22 (1985) 81-104. 